Research Article

\title{
Angle-Resolved Intensity of In-Axis/Off-Axis Polarized Micro-Raman Spectroscopy for Monocrystalline Silicon
}

\author{
Ying Chang, Saisai He, Mingyuan Sun, Aixia Xiao, Jiaxin Zhao, Lulu Ma, and Wei Qiu \\ Tianjin Key Laboratory of Modern Engineering Mechanics, Department of Mechanics, Tianjin University, Tianjin 300350, China \\ Correspondence should be addressed to Wei Qiu; qiuwei@tju.edu.cn
}

Received 9 September 2021; Accepted 18 December 2021; Published 31 December 2021

Academic Editor: Nives Galić

Copyright (c) 2021 Ying Chang et al. This is an open access article distributed under the Creative Commons Attribution License, which permits unrestricted use, distribution, and reproduction in any medium, provided the original work is properly cited.

\begin{abstract}
Monocrystalline silicon (c-Si) is still an important material related to microelectronics/optoelectronics. The nondestructive measurement of the c-Si material and its microstructure is commonly required in scientific research and industrial applications, for which Raman spectroscopy is an indispensable method. However, Raman measurements based on the specific fixed Raman geometry/polarization configuration are limited for the quantified analysis of c-Si performance, which makes it difficult to meet the high-end requirements of advanced silicon-based microelectronics and optoelectronics. Angle-resolved Raman measurements have become a new trend of experimental analysis in the field of materials, physics, mechanics, and optics. In this paper, the characteristics of the angle-resolved polarized Raman scattering of c-Si under the in-axis and off-axis configurations are systematically analyzed. A general theoretical model of the angle-resolved Raman intensity is established, which includes several alterable angle parameters, including the inclination angle, rotation angle of the sample, and polarization directions of the incident laser and scattered light. The diversification of the Raman intensity is given at different angles for various geometries and polarization configurations. The theoretical model is verified and calibrated by typical experiments. In addition, this work provides a reliable basis for the analysis of complex polarized Raman experiments on silicon-based structures.
\end{abstract}

\section{Introduction}

Monocrystalline silicon (c-Si) plays a core role in the advanced manufacturing of optoelectronic and microelectronic devices, with microelectromechanical system (MEMS) being an important semiconductor material $[1,2]$. Raman spectroscopy is one of that commonly used, even necessary, ways to analyze the physical and chemical properties of silicon-based materials and structures. Raman spectroscopy is the result of the nonelastic interaction between the photon of the incidence laser and the phonon of the measured material, which can realize surface or shallow interior characterization. Due to its nondestructive, noncontact, in situ, and highly sensitive characteristics, Raman spectroscopy has been successfully applied in the field of semiconductor materials and two-dimensional materials [3-7].

The Raman spectrum of semiconductor crystals contains structural and physical information, including the crystal state $[8,9]$, crystal plane [10], doping [11], grain size [12], stress/strain [13], Fano resonance [14], and electron mobility [15]. Hence, it is possible to characterize these properties using specific theories of Raman spectroscopy by quantitatively analyzing the wavenumber, intensity, full width at half maximum (FWHM), and symmetry in the Raman spectra from the samples. For instance, using the Raman tensors given by Loudon [16], Anastassakis [17] set up a theoretical model of Raman mechanics. By simplifying this model, de Wolf $[18,19]$ achieved the wavenumber-stress relationship of $\mathrm{c}-\mathrm{Si}$ under the uniaxial or biaxial stress state, which has been widely applied in the stress analyses of semiconductor microstructures made of c-Si [20].

Angle-resolved polarized Raman spectroscopy made it possible to analyze the anisotropy of crystals, such as c-Si, which is closely related to the crystal orientation, polarization, and stress state. Qiu [21] rebuilt the Raman-mechanical theory by regarding the influence of shear stress and provided a method to decouple the in-plane stress 
composites by using angle-resolved polarized Raman spectroscopy. In addition to stress and strain, the crystal plane is also detectable by using angle-resolved polarized Raman spectroscopy. However, it is difficult to obtain sufficient scattering information based on traditional vertical backscatter Raman measurements only. Angle-resolved Raman analyses with different geometric and polarized configurations (namely, in-axis/off-axis angle-resolved polarized Raman spectroscopy) were required to obtain enough information relative to the anisotropic properties of semiconductor materials. In all the existing works, there is a lack of investigations on the Raman intensity under a nonbackscattering configuration. This lack exists because most of the existing Raman spectrometers have vertical backscattering configurations; that is, the incident laser and the scattering light are vertical to the sample surface. The incident laser direction is scarcely adjustable in commercial systems. Self-built off-axial or oblique backscattering systems have been applied to obtain Raman data with nonvertical backscattering configurations and have realized decoupling of the stress components [22] or orientation identification of some crystals [23]. In 2018, Ramabadran [8] systematically analyzed the Raman intensity of c-Si with different cross sections and different polarization states. Several obvious mistakes in the definitions, models, and experiments may be found in that work if read carefully.

In this paper, the theoretical relationship between the Raman intensity and different angles (laser inclination angle, laser polarization direction, and sample rotation angle) is derived and described in detail. The influences of these different angles on the Raman intensity are enumerated and compared, and the significance of an angle change for $\mathrm{c}-\mathrm{Si}$ Raman measurements is expounded. Angle-resolved polarized Raman experiments are carried out, and the experimental results are compared with the theoretical model.

\section{Theoretical Model of the c-Si Raman Intensity}

The definitions of the coordinate systems and angle parameters are shown in Figure 1 to introduce the theoretical model derivation process of this paper in detail, which contains five coordinate systems for the off-axis Raman measurement of c-Si. $X^{\prime} Y^{\prime} Z^{\prime}$ is the incident laser coordinate system, where the $Z^{\prime}$-axis coincides with the incident laser direction. $x^{\prime} y^{\prime} z^{\prime}$ is the scattered light coordinate system, where the $z^{\prime}$-axis coincides with the scattered light direction. $X Y Z$ is the space coordinate system, taken as the reference of the whole experimental system. $x y z$ denotes the sample coordinate system, where $x, y$, and $z$ are the two orthogonal in-plane directions and normal direction of the sample, respectively. [100], [010], and [001] are the crystal directions of the sample in the crystal coordinate system.

The geometrical configuration of the angle-resolved polarized Raman spectrum for a c-Si sample with a random crystal plane is shown in Figure 1, where $\alpha$ is the sample rotation angle, which is the angle between the $x$-axis and the $X$-axis, $\alpha_{\mathrm{I}}$ is the angle between the $x$-axis and the projection of the incident laser onto the measured surface of the sample

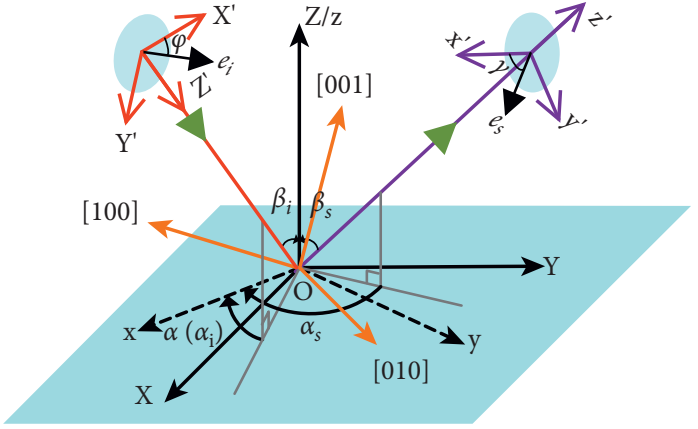

FIgURE 1: Definitions of coordinate systems and angle parameters. The green triangle represents the direction of light propagation; the red line and purple line represent the incident laser and scattering light, respectively. Five coordinate systems are shown in this figure, the incident laser coordinate system $\left(X^{\prime} Y^{\prime} Z^{\prime}\right)$, the scattered light coordinate system $\left(x^{\prime} y^{\prime} z^{\prime}\right)$, the space coordinate system $(X Y Z)$, the sample coordinate system $(x y z)$, and the crystal coordinate system ([100] [010] [001]), respectively.

(without loss of universality, the incident laser was fixed in the $X Z$ plane; hence, $\alpha_{I}=\alpha$ ), $\alpha_{\mathrm{S}}$ is the angle between the $x$ axis and the projection of the scattered light onto the measured surface of the sample, $\beta_{I}$ and $\beta_{S}$ are the inclination angles of the incident laser and scattered light, respectively, and $\varphi$ and $\gamma$ are the polarization directions of the incident laser and scattered light, respectively. The polarization directions of the incident laser and scattered light are expressed as Jones vectors [24].

The model of the polarized Raman intensity for c-Si with an in-axis/off-axis geometric configuration was established based on the general theory as follows. The polarization vectors of the incident laser and scattered light are expressed in the incident laser adaptive coordinate system $\left(X^{\prime} Y^{\prime} Z^{\prime}\right)$ and the scattered light adaptive coordinate system $\left(x^{\prime} y^{\prime} z^{\prime}\right)$ :

$$
\begin{aligned}
& \mathbf{e}_{\mathrm{I}}^{\prime}=\left(\begin{array}{lll}
\cos \varphi & \sin \varphi & 0
\end{array}\right)^{T}, \\
& \mathbf{e}_{s}^{\prime}=\left(\begin{array}{lll}
\cos \gamma & \sin \gamma & 0
\end{array}\right)^{T} .
\end{aligned}
$$

In the sample coordinate system, Jones vectors $\mathbf{e}_{\mathbf{I}}$ and $\mathbf{e}_{\mathbf{S}}$ are obtained based on $\mathbf{e}_{\mathbf{I}}^{\prime}$ and $\mathbf{e}_{S}^{\prime}$ by a coordinate transformation:

$$
\begin{aligned}
& \mathbf{e}_{\mathrm{I}}=\left[\begin{array}{ccc}
\cos \alpha_{\mathrm{I}} & \sin \alpha_{\mathrm{I}} & 0 \\
-\sin \alpha_{\mathrm{I}} & \cos \alpha_{\mathrm{I}} & 0 \\
0 & 0 & 1
\end{array}\right]\left[\begin{array}{ccc}
\cos \beta_{\mathrm{I}} & 0 & \sin \beta_{\mathrm{I}} \\
0 & 1 & 0 \\
-\sin \beta_{\mathrm{I}} & 0 & \cos \beta_{\mathrm{I}}
\end{array}\right]\left[\begin{array}{c}
\cos \varphi \\
\sin \varphi \\
0
\end{array}\right], \\
& \mathbf{e}_{\mathrm{S}}=\left[\begin{array}{ccc}
\cos \alpha_{\mathrm{S}} & \sin \alpha_{\mathrm{S}} & 0 \\
-\sin \alpha_{\mathrm{S}} & \cos \alpha_{\mathrm{S}} & 0 \\
0 & 0 & 1
\end{array}\right]\left[\begin{array}{ccc}
\cos \beta_{\mathrm{S}} & 0 & \sin \beta_{\mathrm{S}} \\
0 & 1 & 0 \\
-\sin \beta_{\mathrm{S}} & 0 & \cos \beta_{\mathrm{S}}
\end{array}\right]\left[\begin{array}{c}
\cos \gamma \\
\sin \gamma \\
0
\end{array}\right] .
\end{aligned}
$$

It is necessary to introduce the optic transform of the airsilicon interface into the theoretical analysis based on repeated experimental verifications and optical notion studies, which is mainly reflected in the influences of refraction and depolarization. The angles $\beta_{\mathrm{I}}$ and $\beta_{\mathrm{S}}$ are modified to $\beta_{\mathrm{I}}^{\prime}$ and $\beta_{\mathrm{S}}^{\prime}$ after refraction, respectively, by using the following equation: 


$$
\begin{aligned}
& \sin \beta_{\mathrm{I}}=n \sin \beta_{\mathrm{I}}^{\prime}, \\
& \sin \beta_{\mathrm{S}}=n \sin \beta_{\mathrm{S}}^{\prime},
\end{aligned}
$$

where $n$ is the refractive index of the air-silicon interface. In addition, the variation in polarization is exemplified mainly in the matrices $\mathbf{F}_{\mathbf{0 1}}$ and $\mathbf{F}_{\mathbf{1 0}}$, which are composed of Fresnel coefficients [25]. $\mathbf{F}_{\mathbf{0 1}}$ and $\mathbf{F}_{\mathbf{1 0}}$ represent the Fresnel matrix of light incident from air to the c-Si and light emitted from the c-Si into the air, respectively:

$$
\begin{aligned}
& \mathbf{F}_{01}=\left[\begin{array}{lll}
t_{01}^{s} & 0 & 0 \\
0 & t_{01}^{p} & 0 \\
0 & 0 & 1
\end{array}\right], \\
& \mathbf{F}_{10}=\left[\begin{array}{lll}
t_{10}^{s} & 0 & 0 \\
0 & t_{10}^{p} & 0 \\
0 & 0 & 1
\end{array}\right], \\
& \text { where }\left\{\begin{array}{l}
t_{01}^{s}=\frac{2 \cos \beta_{\mathrm{I}}}{\cos \beta_{\mathrm{I}}+n \cos \beta_{\mathrm{I}}^{\prime}} \\
t_{01}^{p}=\frac{2 \cos \beta_{\mathrm{I}}}{\cos \beta_{\mathrm{I}}^{\prime}+n \cos \beta_{\mathrm{I}}}, \\
t_{10}^{s}=\frac{2 n \cos \beta_{\mathrm{S}}^{\prime}}{\cos \beta_{\mathrm{S}}+n \cos \beta_{\mathrm{S}}^{\prime}}, \\
t_{10}^{p}=\frac{2 n \cos \beta_{\mathrm{S}}^{\prime}}{\cos \beta_{\mathrm{S}}^{\prime}+n \cos \beta_{\mathrm{S}}}
\end{array}\right.
\end{aligned}
$$

Hence, the polarization vector after refraction and depolarization is shown in the following:

$$
\begin{aligned}
& \mathbf{e}_{\mathrm{I}}=\left[\begin{array}{ccc}
t_{01}^{s} & 0 & 0 \\
0 & t_{01}^{p} & 0 \\
0 & 0 & 1
\end{array}\right]\left[\begin{array}{ccc}
\cos \alpha_{\mathrm{I}} & \sin \alpha_{\mathrm{I}} & 0 \\
-\sin \alpha_{\mathrm{I}} & \cos \alpha_{\mathrm{I}} & 0 \\
0 & 0 & 1
\end{array}\right]\left[\begin{array}{ccc}
\cos \beta_{\mathrm{I}} & 0 & \sin \beta_{\mathrm{I}} \\
0 & 1 & 0 \\
-\sin \beta_{\mathrm{I}} & 0 & \cos \beta_{\mathrm{I}}
\end{array}\right]\left[\begin{array}{c}
\cos \varphi \\
\sin \varphi \\
0
\end{array}\right], \\
& \mathbf{e}_{\mathbf{S}}=\left[\begin{array}{ccc}
t_{10}^{s} & 0 & 0 \\
0 & t_{10}^{p} & 0 \\
0 & 0 & 1
\end{array}\right]\left[\begin{array}{ccc}
\cos \alpha_{\mathrm{S}} & \sin \alpha_{\mathrm{S}} & 0 \\
-\sin \alpha_{\mathrm{S}} & \cos \alpha_{\mathrm{S}} & 0 \\
0 & 0 & 1
\end{array}\right]\left[\begin{array}{ccc}
\cos \beta_{\mathrm{S}} & 0 & \sin \beta_{\mathrm{S}} \\
0 & 1 & 0 \\
-\sin \beta_{\mathrm{S}} & 0 & \cos \beta_{\mathrm{S}}
\end{array}\right]\left[\begin{array}{c}
\cos \gamma \\
\sin \gamma \\
0
\end{array}\right] .
\end{aligned}
$$

The Raman tensor of $\mathrm{c}-\mathrm{Si}$ in the crystal coordinate system is presented as $\mathbf{R}_{\mathbf{j}},(j=1,2,3)$, corresponding to Raman modes along the crystal directions [100], [010], and [001], respectively:

$$
\begin{aligned}
& \mathbf{R}_{1}=\left[\begin{array}{lll}
0 & 0 & 0 \\
0 & 0 & b \\
0 & b & 0
\end{array}\right], \\
& \mathbf{R}_{2}=\left[\begin{array}{lll}
0 & 0 & b \\
0 & 0 & 0 \\
b & 0 & 0
\end{array}\right], \\
& \mathbf{R}_{3}=\left[\begin{array}{lll}
0 & b & 0 \\
b & 0 & 0 \\
0 & 0 & 0
\end{array}\right] .
\end{aligned}
$$

In practical measurement, the Raman tensor should be converted to the sample coordinate system [9] using the rotation matrix A:

$$
\mathbf{A}=\left[\begin{array}{lll}
l_{1} & m_{1} & n_{1} \\
l_{2} & m_{2} & n_{2} \\
l_{3} & m_{3} & n_{3}
\end{array}\right],
$$

where $l_{i}, m_{i}$, and $n_{i}(i=1,2,3)$ are defined as $l_{i}=\cos ([100]$, $\left.x_{i}\right), m_{i}=\cos \left([010], x_{i}\right)$, and $n_{\mathrm{i}}=\cos \left([001], x_{i}\right)$, respectively, and $x_{1}=x, x_{2}=y$, and $x_{3}=z$. Hence, the Raman tensor in the sample coordinate system is as shown as follows:

$$
\mathbf{R}_{\mathbf{x}_{\mathbf{i}}}=\mathbf{A} \mathbf{R}_{\mathbf{j}} \mathbf{A}^{T} \text {. }
$$

According to Raman selection rules, the Raman intensity of each Raman mode in the sample coordinate system is given by

$$
I_{j} \propto\left|\mathbf{e}_{\mathbf{I}} \mathbf{R}_{\mathbf{j}} \mathbf{e}_{\mathbf{S}}\right|^{2}
$$

\section{Angle-Resolved Raman Intensity of (100) c-Si with Typical Geometric Configurations}

Taking the Raman measurement of (100) c-Si under a vertical backscattering configuration (the most commonly used configuration) as an example, the angle-resolved Raman intensity with typical geometric configurations was discussed based on the theoretical model given in Section 2 . A polar coordinate system was used when comparing the Raman intensities of different configurations. A Cartesian coordinate system was used when revealing the distribution of the Raman intensities under each specific configuration.

3.1. Vertical Backscattering $\beta=0^{\circ}$ and $\alpha \in\left[0^{\circ}, 360^{\circ}\right]$. In the vertical backscattering configuration, both the incident laser and the scattered light are normal to the sample surface. It holds that $\alpha_{I}=\alpha_{S}=\alpha$ and $\beta_{I}=\beta_{S}=0^{\circ}$. The intensity of each Raman mode is as follows:

TO ${ }_{1}$ mode: $I_{x} \propto\left|\mathbf{e}_{\mathbf{I}} \mathbf{R}_{\mathbf{x}} \mathbf{e}_{\mathbf{S}}\right|^{2}=0$,

TO ${ }_{2}$ mode: $I_{y} \propto\left|\mathbf{e}_{\mathbf{I}} \mathbf{R}_{\mathbf{y}} \mathbf{e}_{\mathbf{s}}\right|^{2}=0$,

LO mode: $I_{z} \propto\left|\mathbf{e}_{\mathbf{I}} \mathbf{R}_{\mathbf{z}} \mathbf{e}_{\mathbf{S}}\right|^{2}=b^{2} \sin ^{2}(\varphi+\gamma-2 \alpha)$.

As shown in (10), the Raman intensity actually detected under vertical backscattering is shown in Figure 2.

As shown in (10), the Raman intensity actually detected under vertical backscattering is composed only of the information on the LO mode. Moreover, the angle between the polarization direction of the incident laser and that of the scattered light are usually fixed during actual measurement, where $\gamma=\varphi$ is $\mathrm{HH}$ and $\gamma=\varphi+90^{\circ}$ is HV. Hence, in (10), $\alpha$ is mathematically opposite to $\varphi$, which means that the result of rotating the sample rotation angle $\alpha$ is consistent with the inverse rotating polarization direction. In other words, the result of fixed sample rotation angle $\alpha$ and rotated polarization direction $\varphi / \gamma$ is the same as that of fixed $\varphi / \gamma$ and 


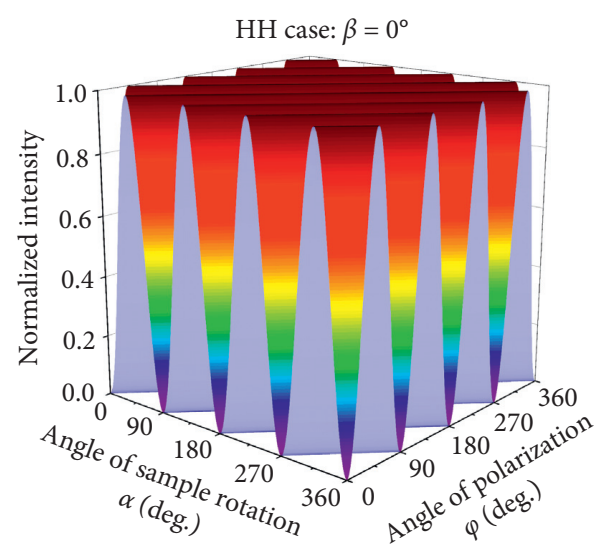

(a)

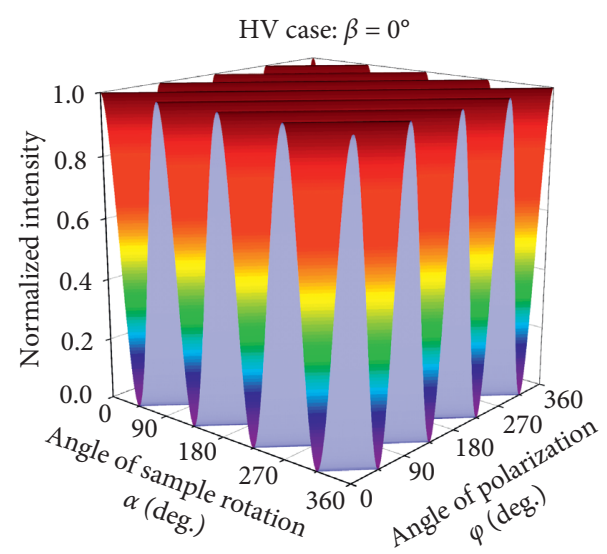

(b)

FIGURE 2: Raman intensity sum distribution of vertical backscattering under different sample rotation angles $\alpha$ and polarization directions $\varphi / \gamma$ of the (a) $\mathrm{HH}$ case and (b) HV case.

rotated $\alpha$. A three-dimensional diagram of the theoretical results according to (10) is shown in Figure 2, which also shows the equivalence of rotating $\alpha$ and $\varphi$.

3.1.1. HH Case. In this case, $\gamma=\varphi$. The Raman intensity of each Raman mode is expressed as (11) when $\alpha$ is fixed but $\varphi$ is rotated $(\varphi=\gamma)$ and as (12) when $\varphi$ is fixed $(\varphi=\gamma)$ but $\alpha$ is rotated. The corresponding changes in the distributions with $\varphi$ are shown in Figure 3(a):

$$
\begin{aligned}
& \text { TO mode: } I_{x} \propto\left|\mathbf{e}_{\mathbf{I}} \mathbf{R}_{\mathbf{x}} \mathbf{e}_{\mathbf{s}}\right|^{2}=0, \\
& \text { TO mode: } I_{y} \propto\left|\mathbf{e}_{\mathbf{I}} \mathbf{R}_{\mathbf{y}} \mathbf{e}_{\mathbf{s}}\right|^{2}=0, \\
& \text { LO mode: } I_{z} \propto\left|\mathbf{e}_{\mathbf{I}} \mathbf{R}_{\mathbf{z}} \mathbf{e}_{\mathbf{S}}\right|^{2}=b^{2} \sin ^{2} 2 \varphi . \\
& \text { TO }_{1} \text { mode: } I_{x} \propto\left|\mathbf{e}_{\mathrm{I}} \mathbf{R}_{\mathbf{x}} \mathbf{e}_{\mathbf{S}}\right|^{2}=0, \\
& \mathrm{TO}_{2} \text { mode: } I_{y} \propto\left|\mathbf{e}_{\mathrm{I}} \mathbf{R}_{\mathbf{y}} \mathbf{e}_{\mathbf{s}}\right|^{2}=0, \\
& \text { LO mode: } I_{z} \propto\left|\mathbf{e}_{\mathbf{I}} \mathbf{R}_{\mathbf{z}} \mathbf{e}_{\mathbf{S}}\right|^{2}=b^{2} \sin ^{2} 2 \alpha .
\end{aligned}
$$

3.1.2. $H V$ Case. In this case, $\gamma=\varphi+90^{\circ}$. The Raman intensity of each Raman mode is expressed as (13) when $\alpha$ is fixed but $\varphi$ is rotated $\left(\gamma=\varphi+90^{\circ}\right)$ and as (14) when $\varphi$ is fixed $\left(\gamma=\varphi+90^{\circ}\right)$ but $\alpha$ is rotated. The corresponding changes in the distributions with $\varphi$ are shown in Figure 3(b):
TO $\mathrm{TOde}_{1} I_{x} \propto\left|\mathbf{e}_{\mathrm{I}} \mathbf{R}_{\mathbf{x}} \mathbf{e}_{\mathrm{S}}\right|^{2}=0$,

TO 2 mode: $I_{y} \propto\left|\mathbf{e}_{\mathbf{I}} \mathbf{R}_{\mathbf{y}} \mathbf{e}_{\mathbf{S}}\right|^{2}=0$,

LO mode: $I_{z} \propto\left|\mathbf{e}_{\mathrm{I}} \mathbf{R}_{\mathbf{z}} \mathbf{e}_{\mathbf{S}}\right|^{2}=b^{2} \cos ^{2} 2 \varphi$,

TO ${ }_{1}$ mode: $I_{x} \propto\left|\mathbf{e}_{\mathbf{I}} \mathbf{R}_{\mathbf{x}} \mathbf{e}_{\mathbf{S}}\right|^{2}=0$,

TO 2 mode: $I_{y} \propto\left|\mathbf{e}_{\mathbf{I}} \mathbf{R}_{\mathbf{y}} \mathbf{e}_{\mathbf{s}}\right|^{2}=0$,

LO mode: $I_{z} \propto\left|\mathbf{e}_{\mathbf{I}} \mathbf{R}_{\mathbf{z}} \mathbf{e}_{\mathbf{S}}\right|^{2}=b^{2} \cos ^{2} 2 \alpha$.

3.1.3. Without an Analyzer $(H H+H V$ Case). In the case of the vertical backscattering configuration without an analyzer, the Raman intensity was the sum of those of the $\mathrm{HH}$ and $\mathrm{HV}$ cases, the distributions of which are shown in Figure 4. Clearly, the Raman intensity is irrelevant to the polarization direction of the incident laser. The $\mathrm{TO}_{1}$ and $\mathrm{TO}_{2}$ modes are Raman invisible of (100) c-Si with vertical backscattering.

3.2. Oblique Backscattering $\beta_{I}=\beta_{S}=\beta>0^{\circ}$ and $\alpha \in\left[0^{\circ}, 360^{\circ}\right]$. In the state of oblique backscattering, $\beta_{I}$ equals $\beta_{S}$ but does not equal 0 . According to the general theory in Section 2, the universal formula of the polarized Raman intensity under the geomatical configuration of oblique backscattering is as follows:

$$
\mathrm{TO}_{1} \text { mode: } I_{x} \propto b^{2} \frac{\left\{\begin{array}{c}
\sin 2 \beta \cos \gamma\left(\cos \alpha \sin \varphi-\cos \varphi \sin \alpha \sqrt{1-\sin ^{2} \beta / 17.23}\right) \\
+8.3 \cos \varphi \sin \beta\left[\begin{array}{c}
\cos \alpha \sin \gamma \sqrt{1-\left(\sin ^{2} \beta / 17.23\right)} \\
+\cos \gamma \sin \alpha\left(\left(\sin ^{2} \beta / 17.23\right)-1\right)
\end{array}\right]
\end{array}\right\}}{\left(\sqrt{17.23-\sin ^{2} \beta}+17.23 \cos \beta\right)^{2}},
$$




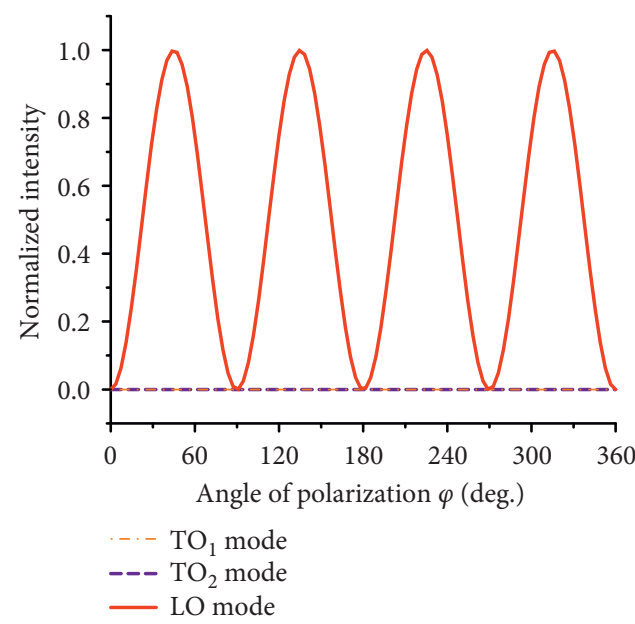

(a)

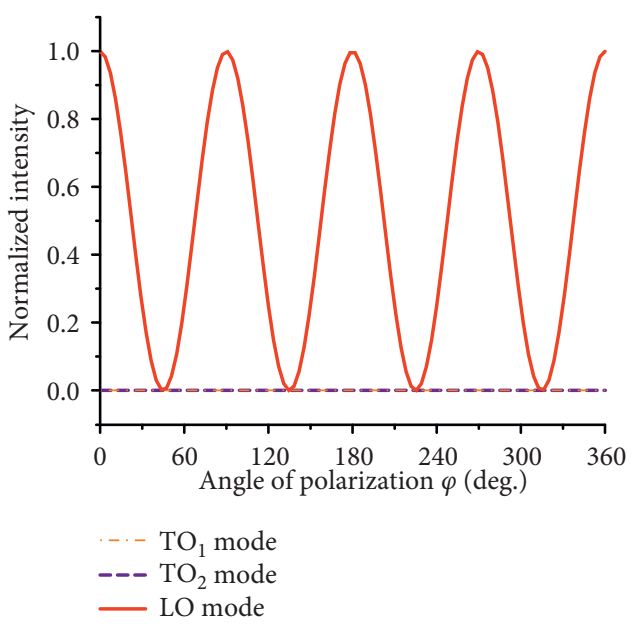

(b)

FIGURE 3: Raman intensity distribution of vertical backscattering for the (a) HH case and (b) HV case.

$$
\begin{aligned}
& \mathrm{TO}_{2} \text { mode: } I_{y} \propto b^{2} \frac{\left\{\begin{array}{c}
\sin 2 \beta \cos \gamma(\sin \alpha \sin \varphi+\cos \alpha \cos \varphi A) \\
+8.3 \cos \varphi \sin \beta\left[\sin \alpha \sin \gamma A-\cos \alpha \cos \gamma\left(\sin ^{2} \beta / 17.23-1\right)\right]
\end{array}\right\}^{2}}{\left(4.15 \cos \beta+4.15 \sqrt{17.23-\sin ^{2} \beta}\right)^{2}}
\end{aligned}
$$

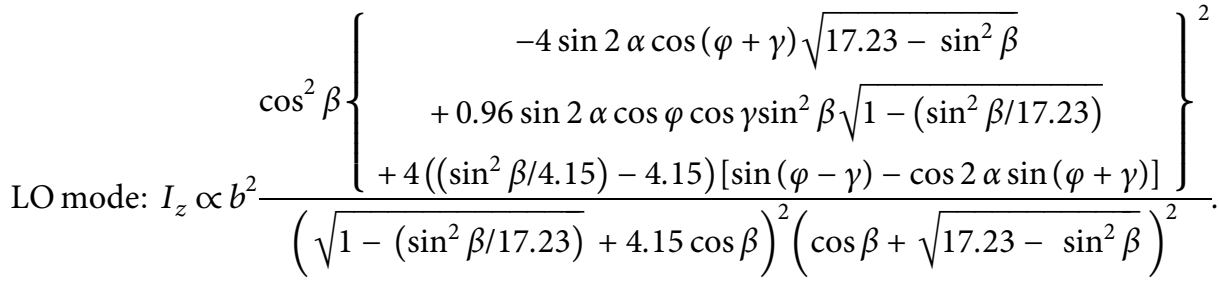

To reveal the influence of the inclination angle $\beta$ on the Raman intensity for different sample rotation angles $\alpha$ and polarization directions $\varphi$, several three-dimensional diagrams are achieved based on (15), as shown in Figures 5 to 7 . Limited by the paper length, only a few typical cases are listed; more information is available in the supporting document. In Figure 5, $\alpha=30^{\circ}, \beta \in\left(0^{\circ}, 90^{\circ}\right)$, and $\varphi \in\left[0^{\circ}, 360^{\circ}\right]$. In Figure $6, \alpha \in\left[0^{\circ}\right.$, $\left.360^{\circ}\right], \beta \in\left(0^{\circ}, 90^{\circ}\right)$, and $\varphi=30^{\circ}$. In Figure $7, \alpha \in\left[0^{\circ}, 360^{\circ}\right]$, $\beta=30^{\circ}$, and $\varphi \in\left[0^{\circ}, 360^{\circ}\right]$. In each figure, the Raman intensity is the sum of the three Raman modes at the corresponding geometrical and polarized configurations. In Figures 5 to 7 , the Raman intensities at different $\alpha, \varphi$, and $\beta$ are obtained by quantifying the effects of refraction and depolarization, where the refractive index of c-Si at $532 \mathrm{~nm}$ is $n=4.151$ [26].

When the polarization direction is fixed but the sample rotation angle is rotated, the trends of the Raman intensity are different from those when the sample rotation angle is fixed but the polarization direction is rotated. Therefore, the result of the change in polarization is different from that of the sample angle rotation. Furthermore, it is obvious that the magnitude of the inclination angle has an effect on the Raman intensity. The trend and period of the change in Raman intensity with the polarization direction and sample rotation angle are different due to the visible TO modes.
3.2.1. $H H$ Case. In this case, $\gamma=\varphi$. The Raman intensity of each Raman mode is given in Figure 8 when the $\alpha$ angle is fixed at $0^{\circ}, 15^{\circ}, 30^{\circ}$, and $45^{\circ}$. In addition, Figure 9 is given to further explore the dissimilar influence of the sample rotation angle and polarization direction on the Raman intensity.

Different from those under the vertical backscattering configuration, the $\mathrm{TO}_{1}$ and $\mathrm{TO}_{2}$ Raman modes are visible (nonzero) under the oblique backscattering configuration. Figure 8 shows that the $\mathrm{TO}_{1}$ and $\mathrm{TO}_{2}$ Raman modes have different trends depending on the polarization direction, and these trends are not similar to one another under the sample rotation angles. The same also goes for the LO. As the sum of $\mathrm{TO}_{1}, \mathrm{TO}_{2}$, and $\mathrm{LO}$, the Raman intensity detected by the spectrometer exhibits several special characteristics, such as periodicity, a maximum and minimum, and phase, which are invisible under vertical backscattering.

3.2.2. HV Case. In this case, $\gamma=\varphi+90^{\circ}$. The trends of the Raman intensities (TO mode, LO mode, and sum intensity) with the change in the polarization direction are shown in Figure 10 when the angle $\alpha$ is fixed at $0^{\circ}, 15^{\circ}, 30^{\circ}$, and $45^{\circ}$. 


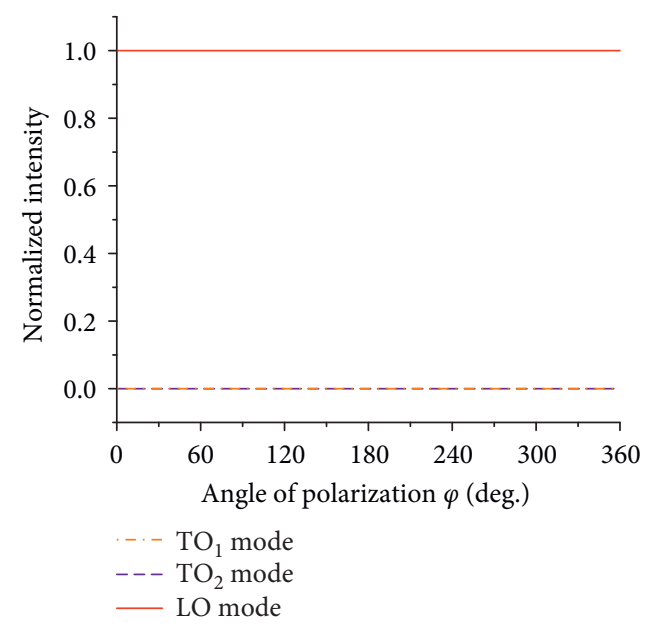

FIGURE 4: Raman intensity distribution of vertical backscattering without an analyzer.

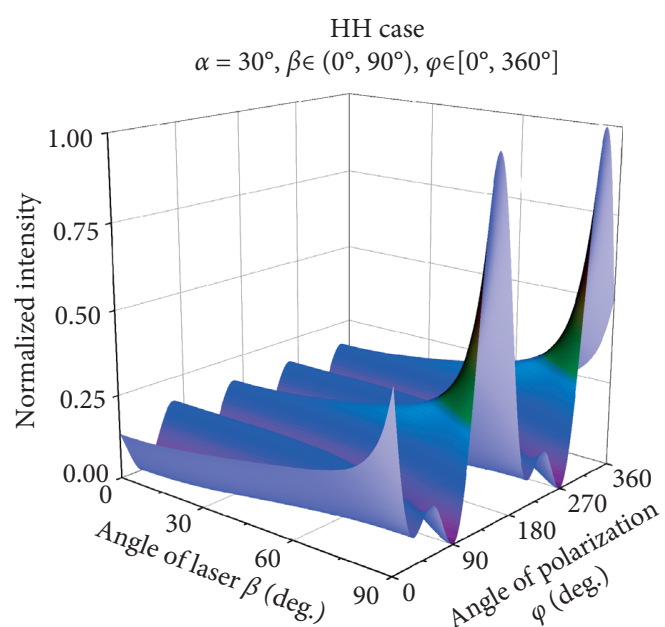

(a)

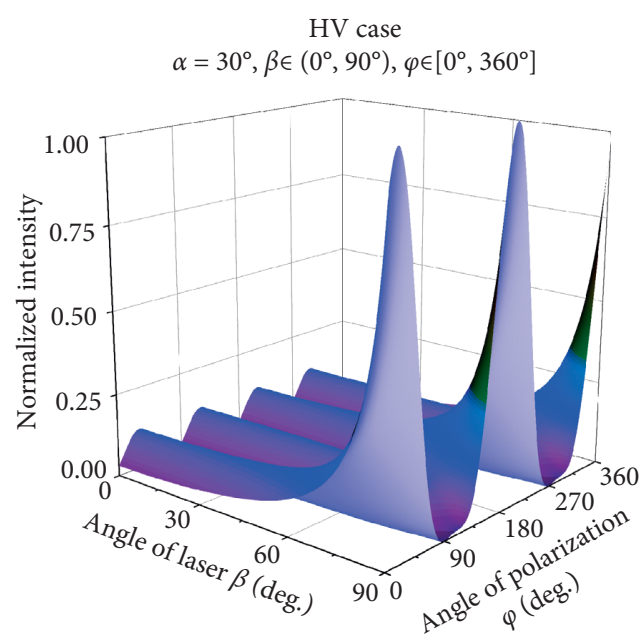

(b)

FIGURE 5: Raman intensity distribution under the oblique backscattering configuration for different inclination angles $\beta$ and polarization directions $\varphi$ when $\alpha=30^{\circ}$ in the (a) HH case and (b) HV case.

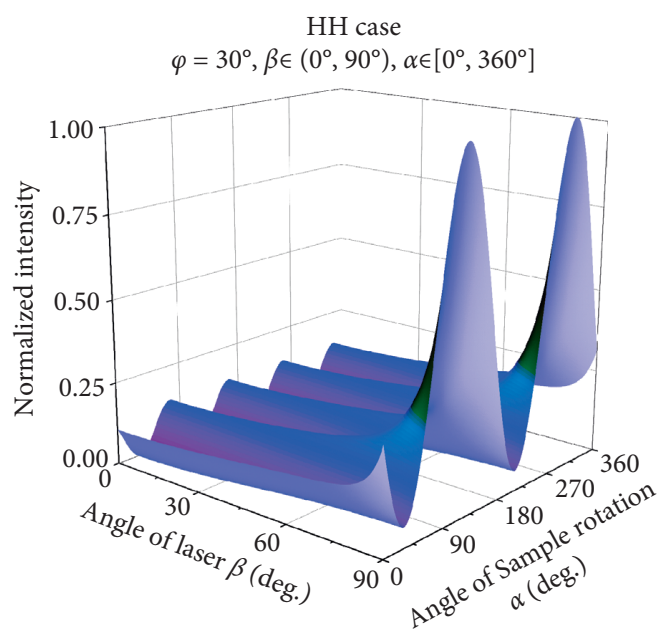

(a)

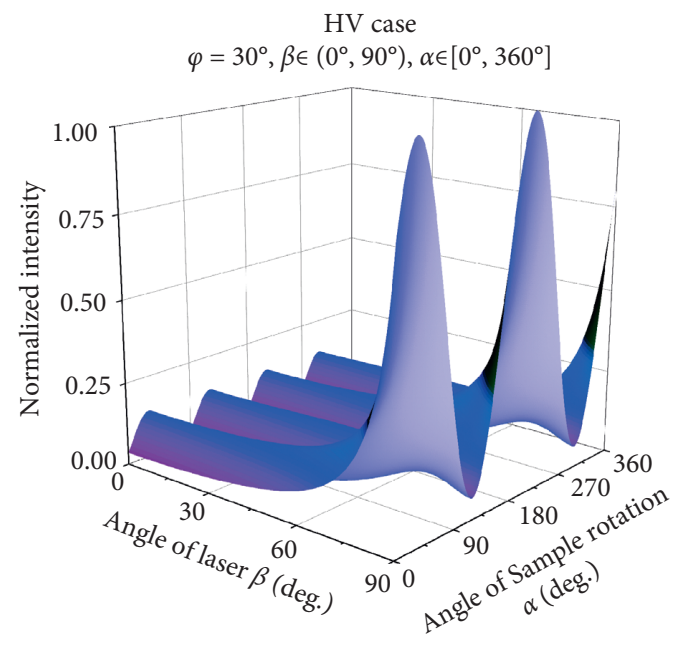

(b)

FIGURE 6: Raman intensity distribution under the oblique backscattering configuration for different inclination angles $\beta$ and sample rotation angles $\alpha$ when $\varphi=30^{\circ}$ in the (a) HH case and (b) HV case. 


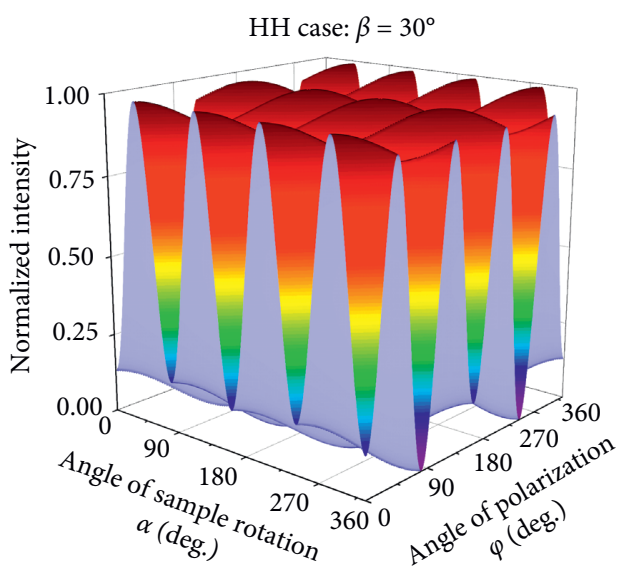

(a)

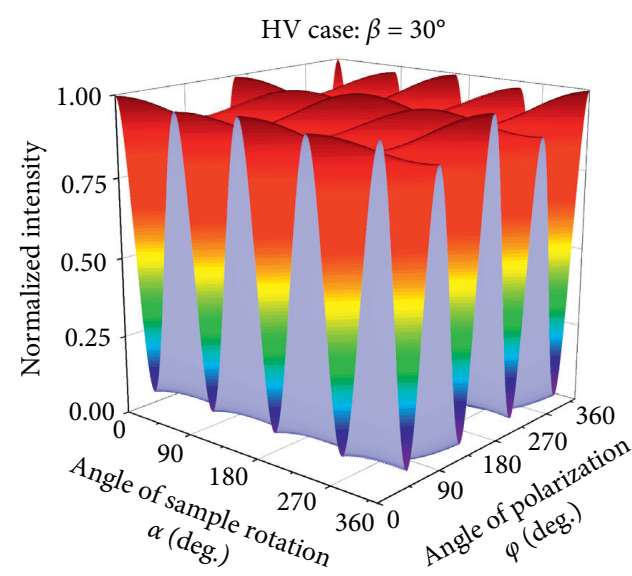

(b)

FiguRE 7: Raman intensity distribution under the oblique backscattering configuration $\left(\beta=30^{\circ}\right)$ for different sample rotation angles $\alpha$ and polarization directions $\varphi$ in the (a) $\mathrm{HH}$ case and (b) HV case.

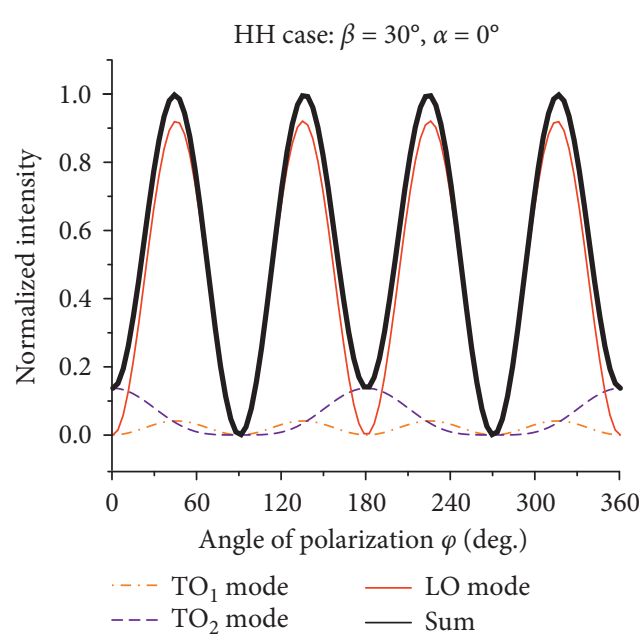

(a)

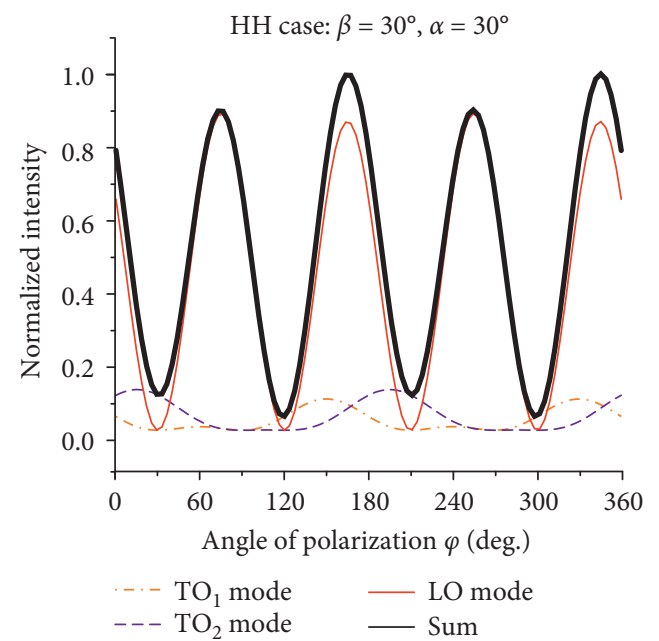

(c)

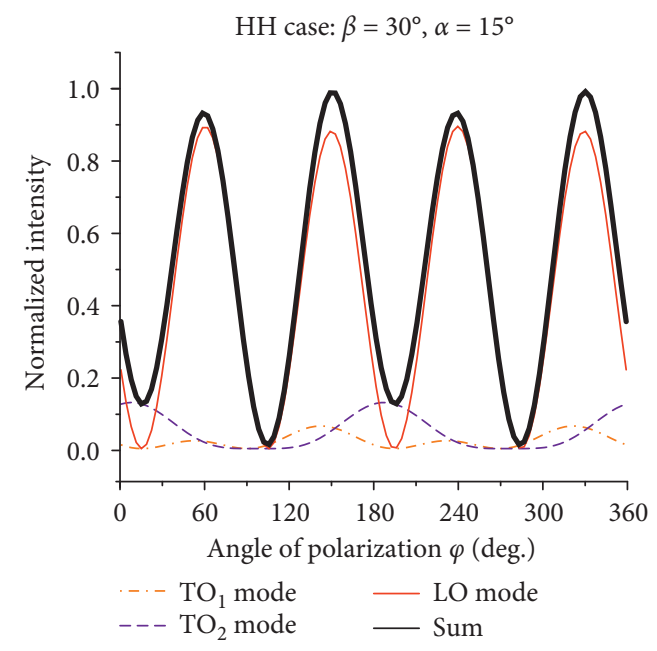

(b)

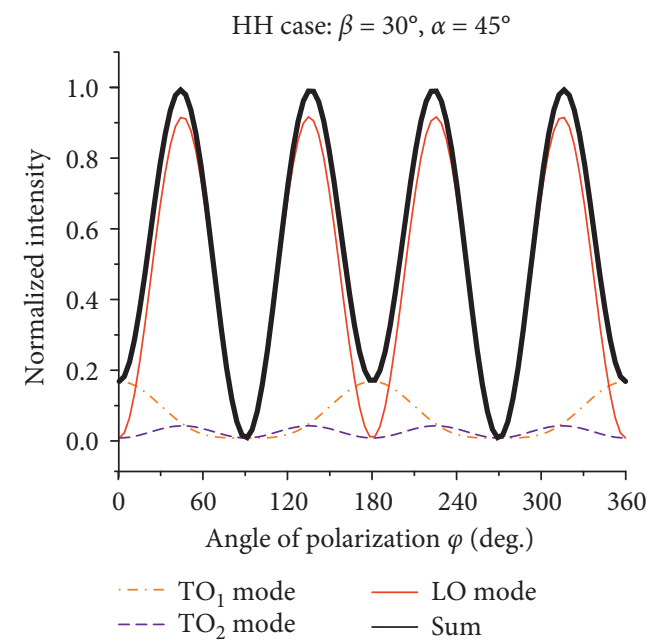

(d)

FIGURE 8: Intensity distributions of Raman modes in the HH case under the oblique backscattering configuration $\left(\beta=30^{\circ}\right)$ for different polarization directions $\varphi$ and sample rotation angles. (a) $\alpha=0^{\circ}$, (b) $\alpha=15^{\circ}$, (c) $\alpha=30^{\circ}$, and (d) $\alpha=45^{\circ}$. 


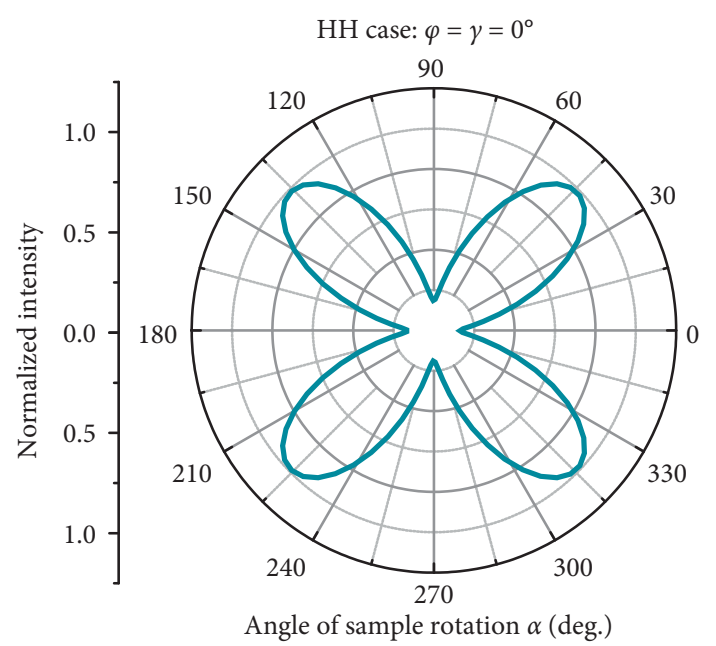

(a)

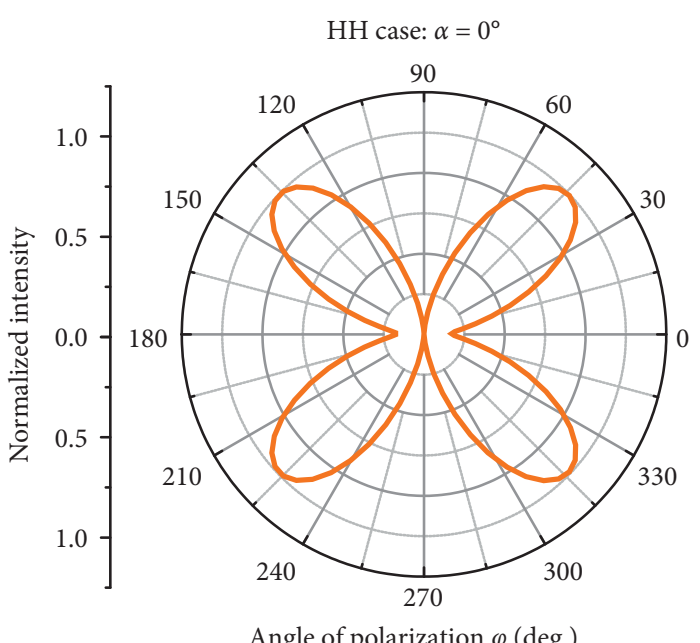

(b)

FIGURE 9: Intensity distributions of Raman modes in the HH case under the oblique backscattering configuration $\left(\beta=30^{\circ}\right)$ for $(\mathrm{a})$ a fixed polarization direction $\varphi=\gamma=0^{\circ}$ but a rotated sample rotation angle $\alpha$ and (b) a fixed sample rotation angle $\alpha=0^{\circ}$ but a rotated polarization direction $\varphi(\gamma=\varphi)$.

Except for the polarization configurations, the angular parameters in Figures 10 and 11 are similar to those in Figures 8 and 9, respectively. By comparing Figure 8 with Figures 10 and 9 and with Figure 11, we see that the Raman intensities for the different polarization configurations vary. In addition, Figure 11 again proves that the result of rotating the sample is not similar to that of rotating the polarization direction under the geometric configuration of oblique backscattering.
3.3. Off-Axis Scattering. $\beta_{\mathrm{I}}>0^{\circ}, \beta_{\mathrm{S}}=0^{\circ}$, and $\alpha \in\left[0^{\circ}, 360^{\circ}\right] \mathrm{An}$ off-axis configuration means that the optical path of the scattering light does not coincide with that of the incident laser. Without loss of universality, $\beta_{\mathrm{I}}=30^{\circ}$ and $\beta_{\mathrm{S}}=0^{\circ}$ in the following analyses of this section. In fact, the results will be the same if $\beta_{\mathrm{I}}=0^{\circ}$ and $\beta_{\mathrm{S}}=30^{\circ}$. According to the general theory in Section 2, the universal formula of the polarized Raman intensity under the geomatical configuration of offaxis backscattering is as follows:

$$
\begin{aligned}
& \text { TO mode: } I_{x} \propto b^{2} \frac{\cos ^{2} \varphi \sin ^{2} \beta \sin ^{2}(\gamma-\alpha)}{17.23}, \\
& \text { TO mode: } I_{y} \propto b^{2} \frac{\cos ^{2} \varphi \sin ^{2} \beta \cos ^{2}(\alpha-\gamma)}{17.23}, \\
& \text { LO mode: } I_{z} \propto b^{2} \cos ^{2} \beta\left(\begin{array}{l}
\frac{\cos (\alpha-\gamma)\left(2 \cos \alpha \sin \varphi-2 \cos \varphi \sin \alpha \sqrt{1-\left(\sin ^{2} \beta / 17.23\right)}\right)}{\left(\sqrt{1-\left(\sin ^{2} \beta / 17.23\right)}+4.15 \cos \beta\right)} \\
\frac{\sin (\gamma-\alpha)\left(2 \sin \alpha \sin \varphi+2 \cos \alpha \cos \varphi \sqrt{1-\left(\sin ^{2} \beta / 17.23\right)}\right.}{+\left(\cos \beta+\sqrt{17.23-\sin ^{2} \beta}\right)}
\end{array}\right) .
\end{aligned}
$$

To reveal the influence of the inclination angle $\beta_{\mathrm{I}}$ on the Raman intensity under different sample rotation angles $\alpha$ and polarization directions $\varphi$, several three-dimensional diagrams are developed based on the general theory given in Section 2, as shown in Figure 12. The above relationships are achieved by taking into account the effects of refraction and depolarization, where the refractive index of c-Si at $532 \mathrm{~nm}$ is $n=4.151$ [26]. It must be noted that the theoretical result of off-axis scattering is simpler than that of oblique backscattering, but it is difficult to achieve from the device aspect [22]. 


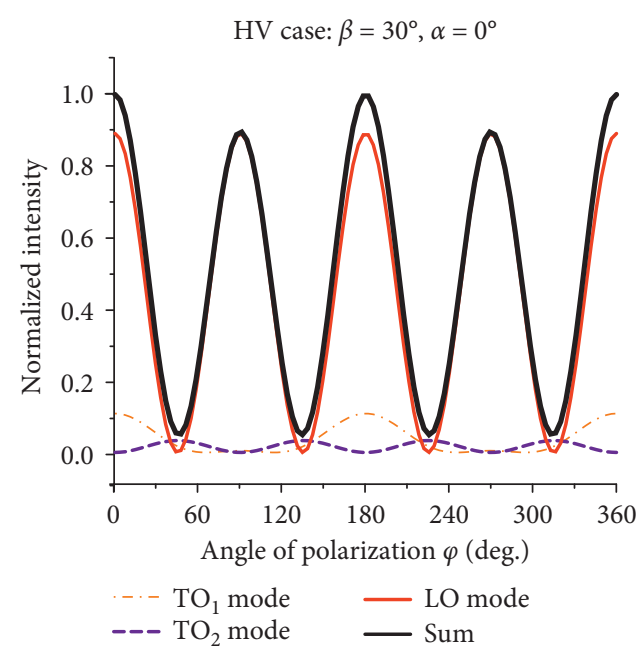

(a)

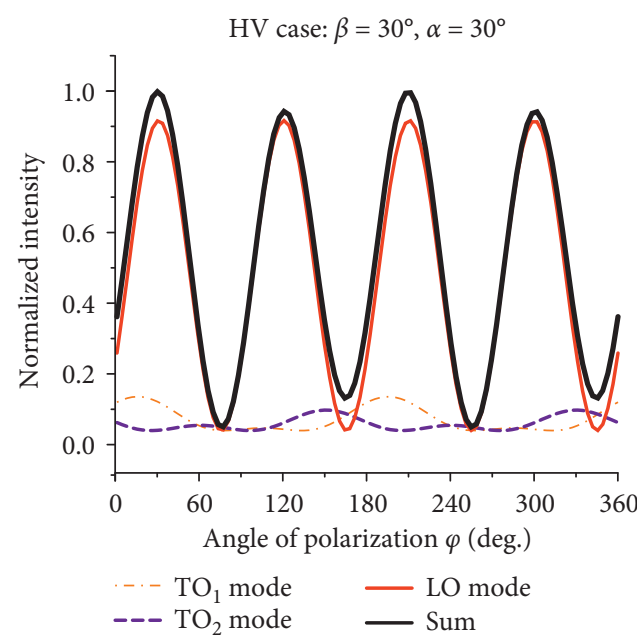

(c)

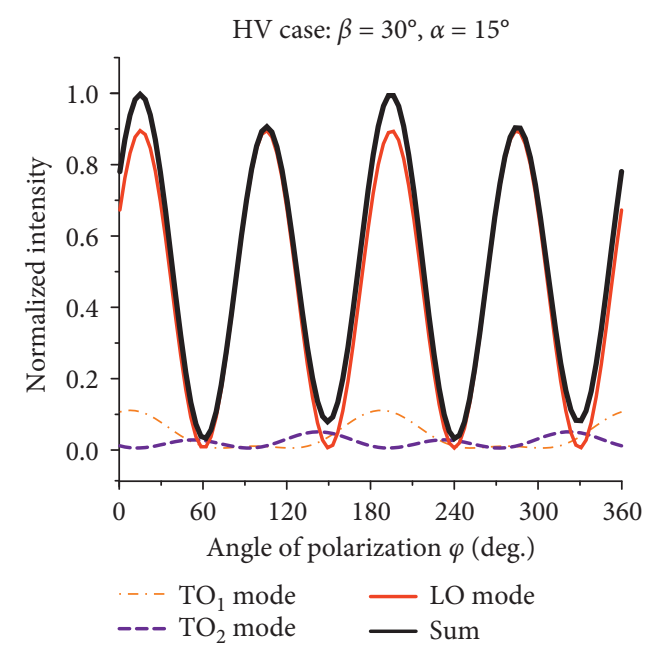

(b)

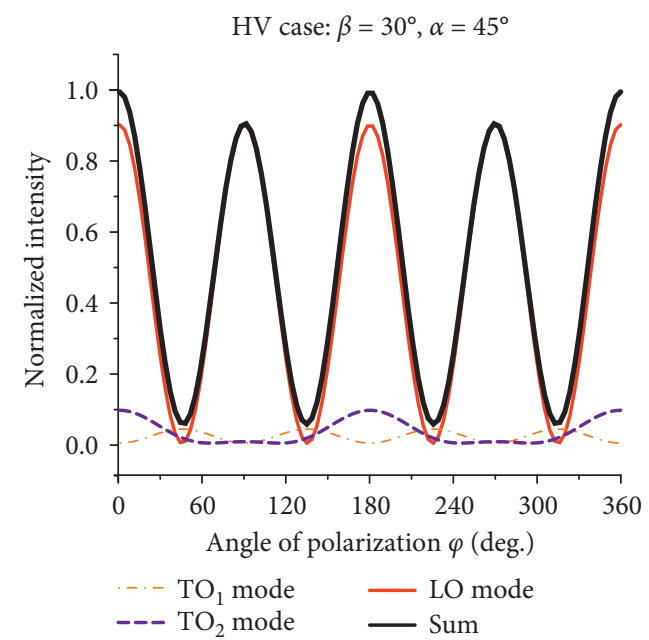

(d)

FIgURE 10: Intensity distributions of Raman modes in the HV case under the oblique backscattering configuration $\left(\beta=30^{\circ}\right)$ for different polarization directions $\varphi$ and sample rotation angles: (a) $\alpha=0^{\circ}$, (b) $\alpha=15^{\circ}$, (c) $\alpha=30^{\circ}$, and (d) $\alpha=45^{\circ}$.

3.3.1. HH Case. In this case, $\gamma=\varphi$. The Raman intensity of each Raman mode is given in Figure 13 when the angle $\alpha$ is fixed at $0^{\circ}$ and $30^{\circ}$.

3.3.2. $H V$ Case. In this case, $\gamma=\varphi+90^{\circ}$. The trends of the Raman intensities (TO mode, LO mode, and sum intensity) with the polarization direction are shown in Figure 14 when the angle $\alpha$ is fixed at $0^{\circ}$ and $30^{\circ}$.

\section{Angle-Resolved Raman Intensity of (110) c-Si}

In the state of the (110) plane, the $x$-axis is $[-110]$, the $y$-axis is [001], and the $z$-axis is [110] in Figure 1. The Raman tensor in the sample coordinate system is obtained from (6)-(8), as shown in the following:

$$
\begin{aligned}
& \mathbf{R}_{1}=\frac{\sqrt{2}}{2} b\left[\begin{array}{ccc}
0 & 1 & 0 \\
1 & 0 & 1 \\
0 & 1 & 0
\end{array}\right] \\
& \mathbf{R}_{2}=\frac{\sqrt{2}}{2} b\left[\begin{array}{ccc}
0 & -1 & 0 \\
-1 & 0 & 1 \\
0 & 1 & 0
\end{array}\right]
\end{aligned}
$$

$$
\mathbf{R}_{3}=b\left[\begin{array}{ccc}
-1 & 0 & 0 \\
0 & 0 & 0 \\
0 & 0 & 1
\end{array}\right]
$$




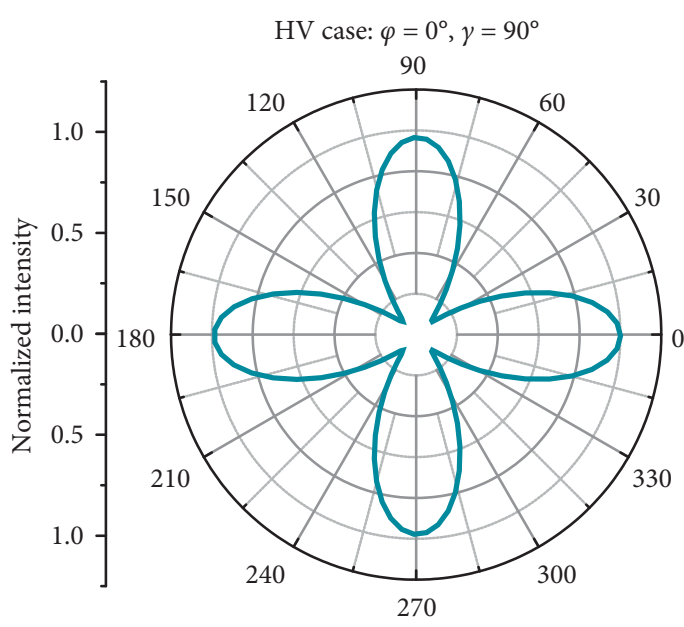

Angle of sample rotation $\alpha$ (deg.)

(a)

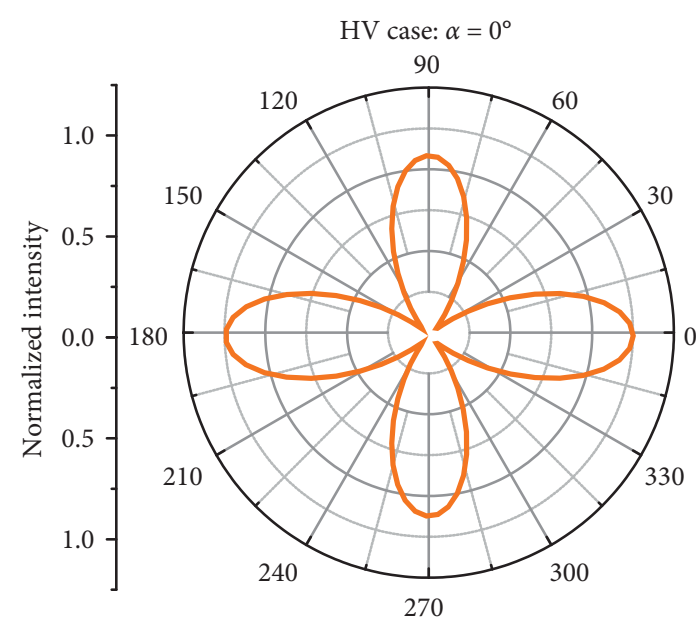

Angle of polarization $\varphi$ (deg.)

(b)

FIGURE 11: Intensity distributions of Raman modes in the HV case under the oblique backscattering configuration $\left(\beta=30^{\circ}\right)$ for (a) a fixed polarization direction $\varphi=0^{\circ}$ but a rotated sample rotation angle $\alpha$ and (b) a fixed sample rotation angle $\alpha=0^{\circ}$ but a rotated polarization direction $\varphi\left(\gamma=\varphi+90^{\circ}\right)$.

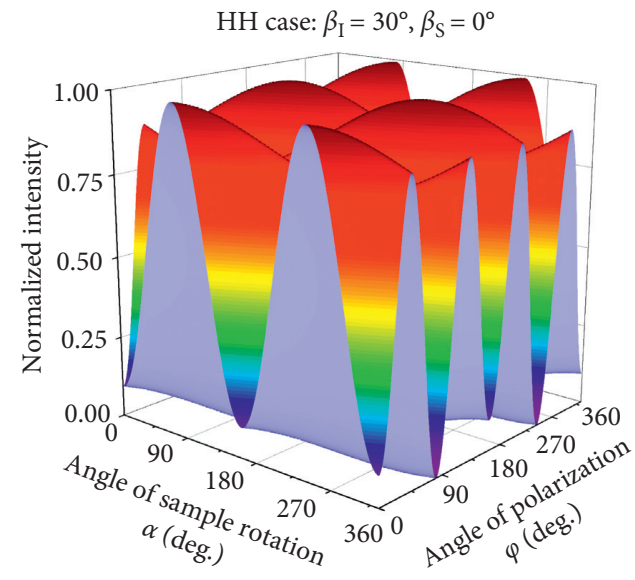

(a)

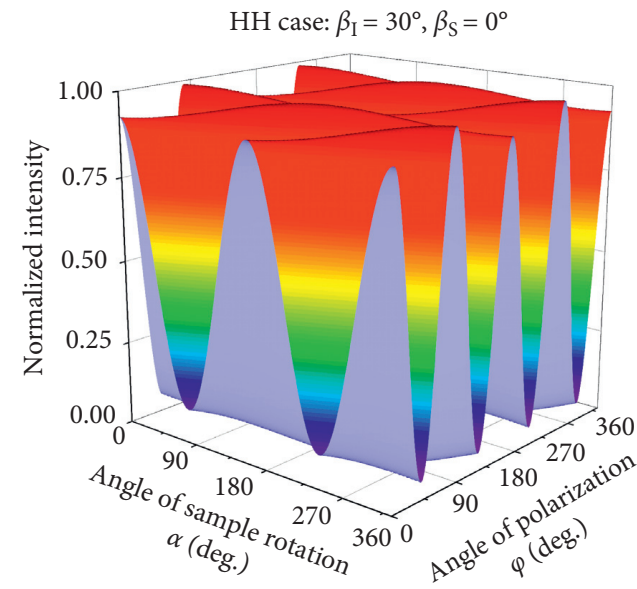

(b)

FIGURE 12: Raman intensity distribution under off-axis scattering $\left(\beta_{\mathrm{I}}=30^{\circ}, \beta_{\mathrm{S}}=0^{\circ}\right)$ for different sample rotation angles $\alpha$ and polarization directions $\varphi$ in the (a) $\mathrm{HH}$ case and (b) HV case.

In the HH case, $\varphi=\gamma$. The universal formula of the polarized Raman intensity is presented in (18)-(20), where $\beta_{\mathrm{I}}=\beta_{\mathrm{S}}=0^{\circ}$ during vertical backscattering, $\beta_{\mathrm{I}}=\beta_{\mathrm{S}}=30^{\circ}$ during oblique backscattering, and $\beta_{\mathrm{I}}=30^{\circ}$ and $\beta_{\mathrm{S}}=0^{\circ}$ during off-axis scattering. In the above configurations, the trends of the Raman intensities with changes in the polarization are shown in Figure 15. Consider

$\mathrm{TO}_{1}$ mode: $I_{x} \propto \frac{1}{2} b^{2} \sin ^{2}(2 \alpha-2 \varphi)$,

$\mathrm{TO}_{2}$ mode: $I_{y} \propto \frac{1}{2} b^{2} \sin ^{2}(2 \alpha-2 \varphi)$,

LO mode: $I_{z} \propto b^{2} \cos ^{4}(\alpha-\varphi)$. 


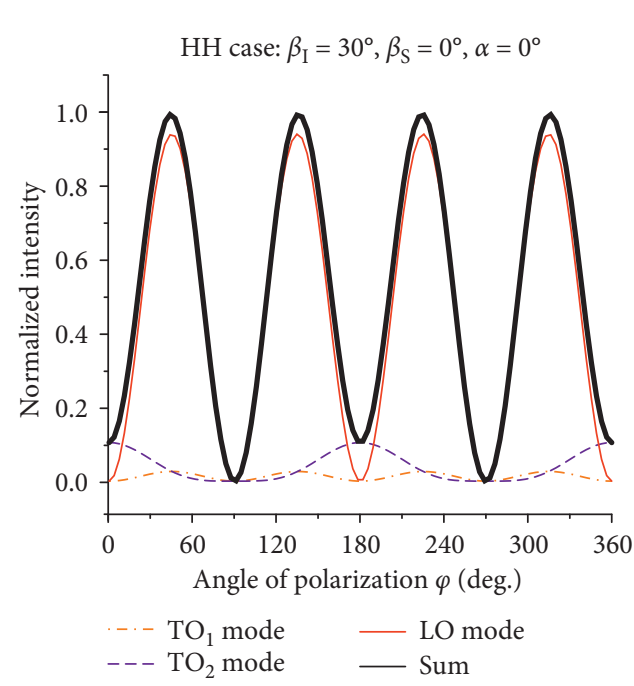

(a)

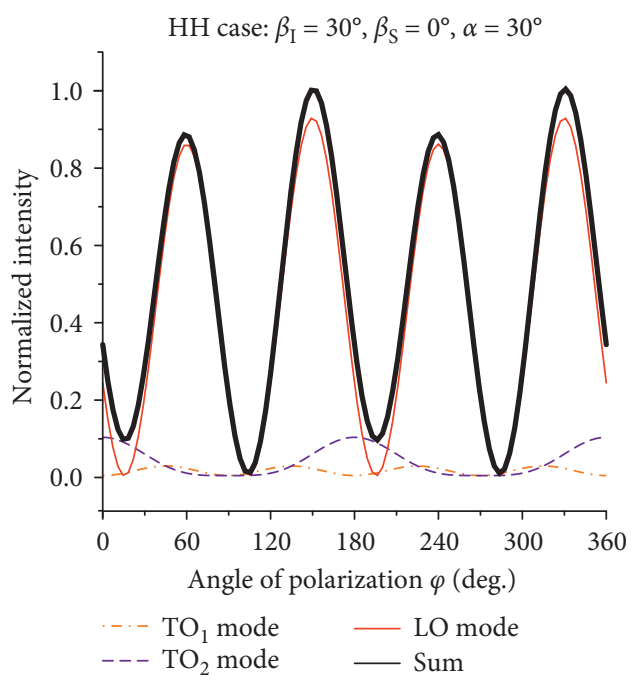

(b)

FIgURE 13: Intensity distributions of Raman modes in the HH case under off-axis scattering $\left(\beta_{\mathrm{I}}=30^{\circ}, \beta_{\mathrm{S}}=0^{\circ}\right)$ for different polarization directions $\varphi$ and sample rotation angles: (a) $\alpha=0^{\circ}$ and (b) $\alpha=30^{\circ}$.

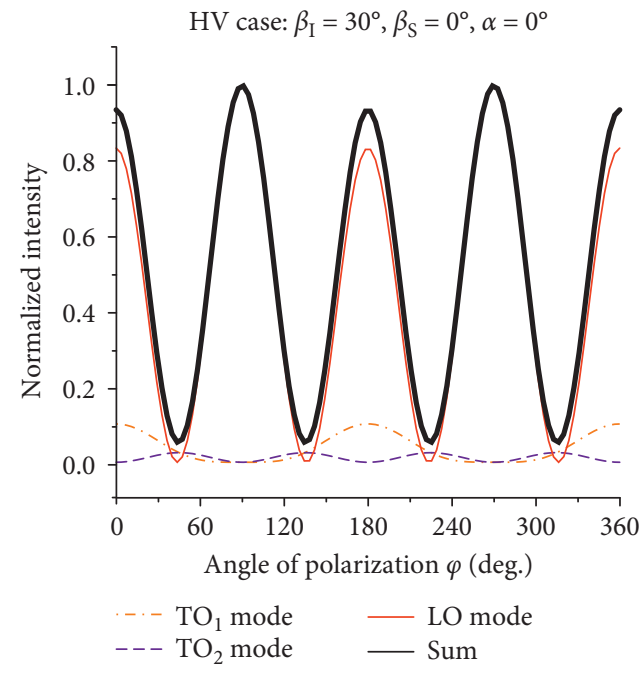

(a)

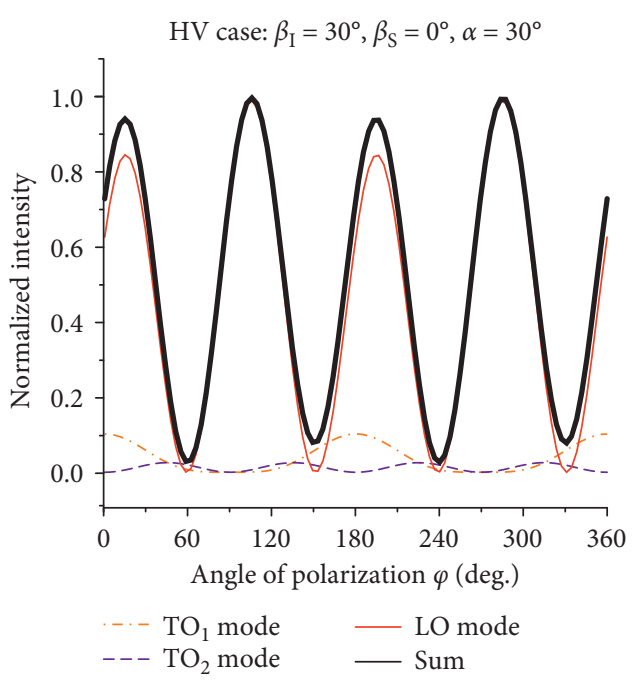

(b)

FIgURE 14: Intensity distributions of Raman modes in the HV case under off-axis scattering $\left(\beta_{\mathrm{I}}=30^{\circ}, \beta_{\mathrm{S}}=0^{\circ}\right)$ for different polarization directions $\varphi$ and sample rotation angles: (a) $\alpha=0^{\circ}$ and (b) $\alpha=30^{\circ}$.

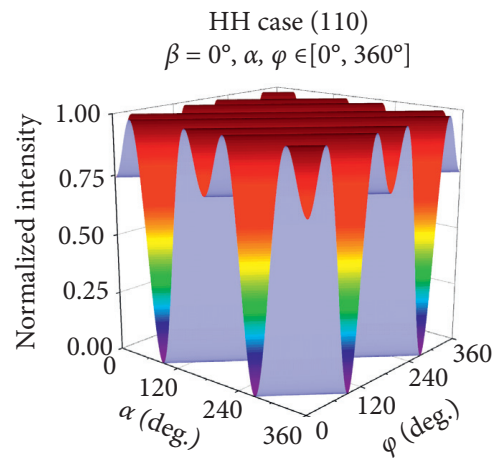

(a)

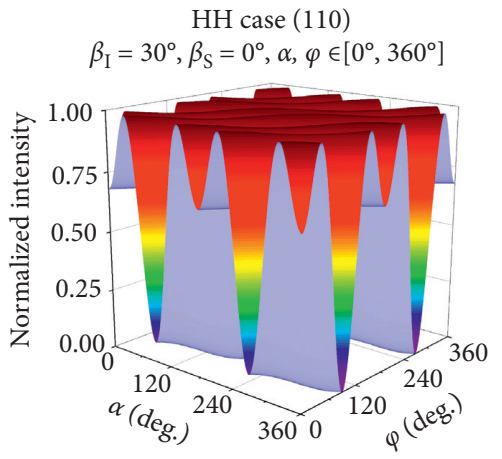

(b)

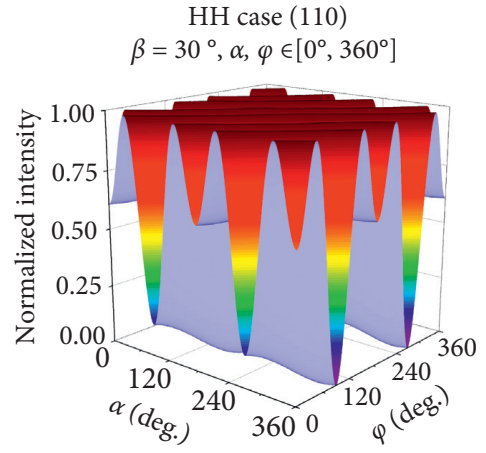

(c)

FIGURE 15: Intensity distributions of Raman modes of (110) c-Si in the HH case for different polarization directions $\varphi$ and sample rotation angles $\alpha$ under (a) vertical backscattering $\left(\beta=0^{\circ}\right)$, (b) off-axis scattering $\left(\beta=30^{\circ}\right)$, and (c) oblique backscattering $\left(\beta_{\mathrm{I}}=30^{\circ}, \beta_{\mathrm{S}}=0^{\circ}\right)$. 
Then, consider

$\mathrm{TO}_{1}$ mode $: I_{x} \propto b^{2}\left[\begin{array}{c}\frac{11.74 \sin 2 \alpha \cos \beta \sqrt{1-\sin ^{2} \beta / 17.23}\left(0.058 \sin ^{2} \beta \cos ^{2} \varphi-\cos 2 \varphi\right)}{\left(\sqrt{1-\sin ^{2} \beta / 17.23}+4.15 \cos \beta\right)\left(\cos \beta+\sqrt{17.23-\sin ^{2} \beta}\right)} \\ -\left(1.41 \sqrt{1-\sin ^{2} \beta / 17.23}+0.34 \cos \beta\right) \frac{\cos \alpha \sin \varphi}{\left(\sqrt{1-\sin ^{2} \beta / 17.23}+4.15 \cos \beta\right)} \cos \varphi \sin \beta\end{array}\right]^{2}$

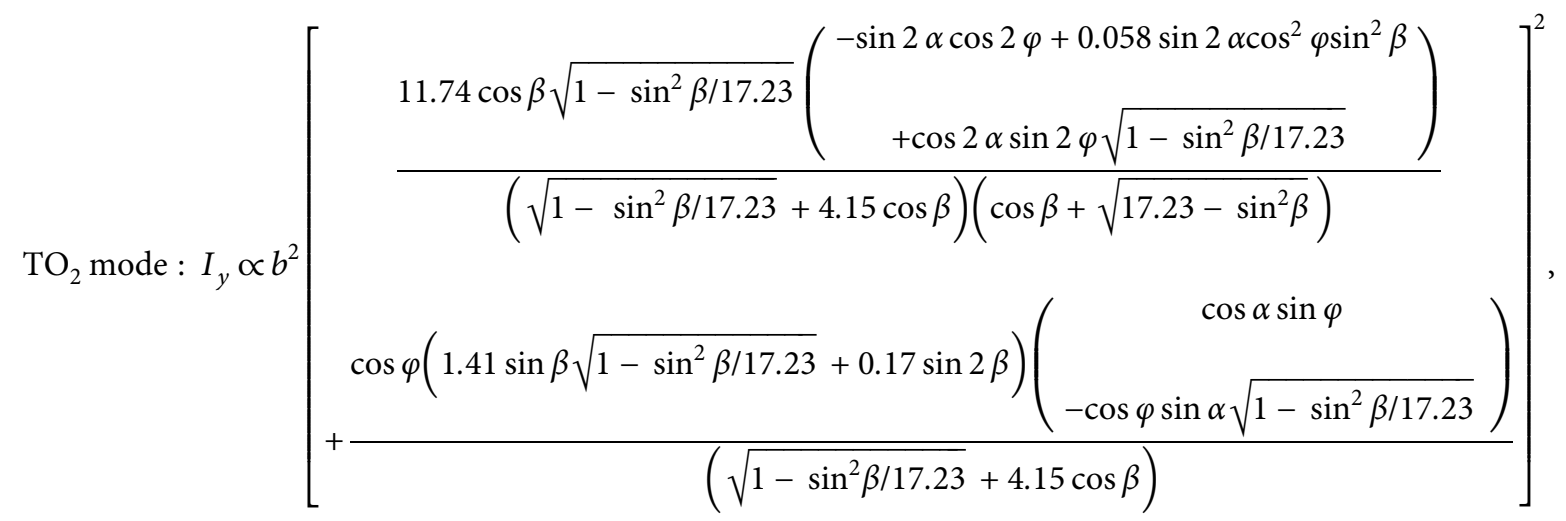

LO mode $: I_{z} \propto b^{2}\left[\frac{16.6 \cos \beta \sqrt{1-\sin ^{2} \beta / 17.23}}{\left(\cos \beta+\sqrt{17.23-\sin ^{2} \beta}\right)^{2}}\left(\begin{array}{c}+\sin ^{2} \alpha \sin ^{2} \varphi+\cos ^{2} \alpha \cos ^{2} \varphi \\ -0.058 \cos ^{2} \alpha \cos ^{2} \varphi \sin ^{2} \beta \\ +0.5 \sin 2 \alpha \sin 2 \varphi \sqrt{1-\sin ^{2} \beta / 17.23}\end{array}\right]^{2}-0.058 \cos ^{2} \varphi \sin ^{2} \beta\right]^{2}$

$\mathrm{TO}_{1}$ mode $I_{x} \propto b^{2}\left[\begin{array}{c}\frac{1.41 \cos \beta \sin (\varphi-\alpha)\left(\sin \alpha \sin \varphi+\cos \alpha \cos \varphi \sqrt{1-\left(\sin ^{2} \beta / 17.23\right)}\right)}{\left(\cos \beta+\sqrt{17.23-\sin ^{2} \beta}\right)}-0.17 \sin (\varphi-\alpha) \cos \varphi \sin \beta \\ +\frac{1.41 \cos \beta \cos (\varphi-\alpha)\left(\cos \alpha \sin \varphi-\cos \varphi \sin \alpha \sqrt{1-\left(\sin ^{2} \beta / 17.23\right)}\right)}{\left(\sqrt{1-\left(\sin ^{2} \beta / 17.23\right)}+4.15 \cos \beta\right)}\end{array}\right]^{2}$

$\mathrm{TO}_{2}$ mode $I_{y} \propto b^{2}\left[\begin{array}{c}\frac{1.41 \cos \beta \sin (\varphi-\alpha)\left(\sin \alpha \sin \varphi+\cos \alpha \cos \varphi \sqrt{1-\left(\sin ^{2} \beta / 17.23\right)}\right)}{\left(\cos \beta+\sqrt{17.23-\sin ^{2} \beta}\right)}-0.17 \sin (\varphi-\alpha) \cos \varphi \sin \beta \\ +\frac{1.41 \cos \beta \cos (\varphi-\alpha)\left(\cos \alpha \sin \varphi-\cos \varphi \sin \alpha \sqrt{1-\left(\sin ^{2} \beta / 17.23\right)}\right)}{\left(\sqrt{1-\left(\sin ^{2} \beta / 17.23\right)}+4.15 \cos \beta\right)}\end{array}\right]^{2}$

LO mode : $I_{z} \propto b^{2} 4 \cos ^{2} \beta \cos ^{2}(\alpha-\varphi) \frac{\left(\sin \alpha \sin \varphi+\cos \alpha \cos \varphi \sqrt{1-\left(\sin ^{2} \beta / 17.23\right)}\right)^{2}}{\left(\cos \beta+\sqrt{17.23-\sin ^{2} \beta}\right)^{2}}$. 


\section{Experiment and Result Discussion}

5.1. Comparison of the Experiment and Theory. The Raman intensities are measured under the geometric configurations of vertical backscattering and oblique backscattering to verify the correctness of the theoretical analyses. All experiments were performed using a self-built angle-resolved polarization Raman system.

With a $532 \mathrm{~nm}$ laser and a $50 \times($ numerical aperture 0.42$)$ lens, the self-built angle-resolved polarization Raman system was developed as shown in Figure 16. Different from the traditional micro-Raman spectroscopy, this system was built based on an oblique backscattering structure, integrated with the controls of polarization, inclination, and sample rotation. In this system, all the angle parameters, including the inclination angle of the optic path, in situ rotation angle of sample, incident polarization angle, and scattering polarization angle, were adjustable and controllable. A doublepolished (100) c-Si wafer was used as the sample, which is provided and polished by Fangdao Semiconductor (Guangzhou Fangdao Semiconductor Co., Ltd., Guangzhou, Guangdong, China). Ten random sampling spots were detected under each polarized and geometrical configuration. All the experiments used the same sampling time of $3 \mathrm{~s}$, the laser power which was $150 \mathrm{~mW}$, and an $1800 \mathrm{l} / \mathrm{mm}$ grating.

Figure 17 shows that most of the experimental points (black solid points) are identical to the theoretical results (red solid line) except for a few. The reason that a few experimental points deviate from the theoretical results includes the angle control error of polarization and the experimental environment.

5.2. Problems in Previous Works. There were few works on the angle-resolved polarized Raman intensity under different geometrical configurations until the work of Ramabadran and Roughani [8] was presented. In this work, the Raman intensity of $\mathrm{c}$-Si using in-axis/off-axis polarized Raman spectroscopy was analyzed by calculating the variation in Raman intensity with the incident laser direction, scattering direction, polarization direction, and sample rotation angle. However, some core errors have been found in the published version, including inconsistent context definitions, incorrect concept definitions, and some experimental results that conflict with the corresponding theoretical results, which were discussed in Appendix A.

5.3. The Effect of the Air-Silicon Interface on the Laser. The theoretical Raman intensities under vertical backscattering were used without consideration of the effect of refraction and depolarization of (100) $\mathrm{c}-\mathrm{Si}$ in the previous work. However, under the oblique backscattering and offaxis scattering configurations, the influences of refraction and depolarization become nonnegligible. Figure 18 [26] indicates the refractive index of the air-silicon interface for different incident laser wavelengths. The laser used in the experiments of this work has a wavelength $532 \mathrm{~nm}$; hence, the corresponding refractive index of the air-silicon interface $n=4.151$ [26], which is mainly associated with the

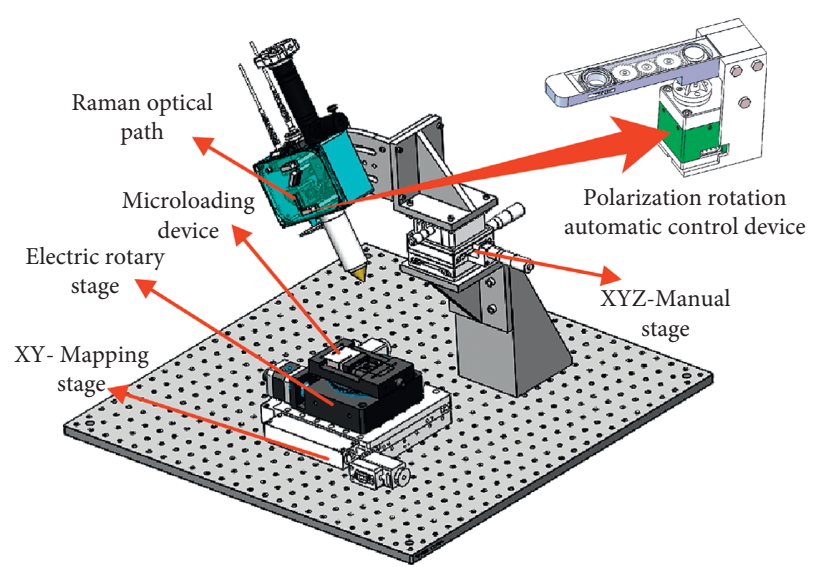

FIGURE 16: Assembly diagram of self-built angle-resolved polarization Raman system.

magnitude of the refractive index $n$. The following shows that the value of $n$ is too large to ignore.

Based on the theoretical model above, (20) and (21) give the Raman intensities under the oblique backscattering configuration $\left(\beta=30^{\circ}\right)$ in the $\mathrm{HH}$ and $\mathrm{HV}$ cases, respectively. In these two equations, the subscripts NR and ND denote the influence of the refractive index and depolarization, respectively, which were not considered; hence, the equation without the subscripts $\mathrm{NR}$ and $\mathrm{ND}$ is the result considering the influence of the refractive index and depolarization. According to (20) and (21), Figure 19 shows the distribution of the Raman intensity with the polarization direction, which clearly indicates that neither refraction nor depolarization is ignorable in the angle-resolved Raman measurement:

$$
\begin{aligned}
& I_{\mathrm{NRND}} \propto \sin ^{2} 2 \varphi+0.75 \cos ^{4} \varphi, \\
& I_{\mathrm{ND}} \propto \sin ^{2} 2 \varphi+0.06 \cos ^{4} \varphi, \\
& I \propto 0.40 \sin ^{2} 2 \varphi+0.06 \cos ^{4} \varphi, \\
& I_{\mathrm{NRND}} \propto \cos ^{2} 2 \varphi+0.75 \sin ^{2} \varphi \cos ^{2} \varphi, \\
& I_{\mathrm{ND}} \propto \cos ^{2} 2 \varphi+0.06 \sin ^{2} \varphi \cos ^{2} \varphi, \\
& I \propto 0.38 \cos ^{2} 2 \varphi+0.06 \sin ^{2} \varphi \cos ^{2} \varphi \\
& +0.01(1+1.53 \cos 2 \varphi)^{2} .
\end{aligned}
$$

5.4. Effect of the Numerical Aperture on the Raman Intensity. In the above measurement, the incident laser and scattered light are considered collimated beams.

However, the incident laser focused on the sample after passing through the microscope lens has the shape of a taper or cone, and the sine of the cone angle is defined as the numerical aperture (NA). Some published works found that the influence of the NA could be ignored when $\mathrm{NA} \leq 0.4$. However, when N.A. $>0.4$, the effect of the NA on the Raman intensity must be considered.

The Raman intensity is actually the sum of all the scattering light in the range of the optic cone from the materials of the sample that is excited by the incident laser 


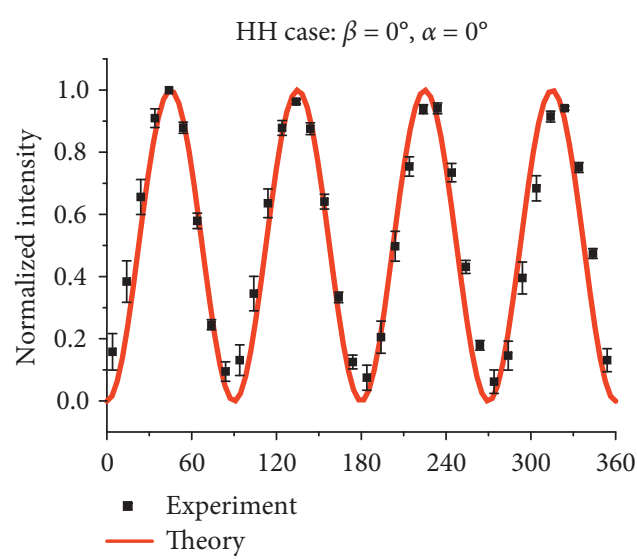

(a)

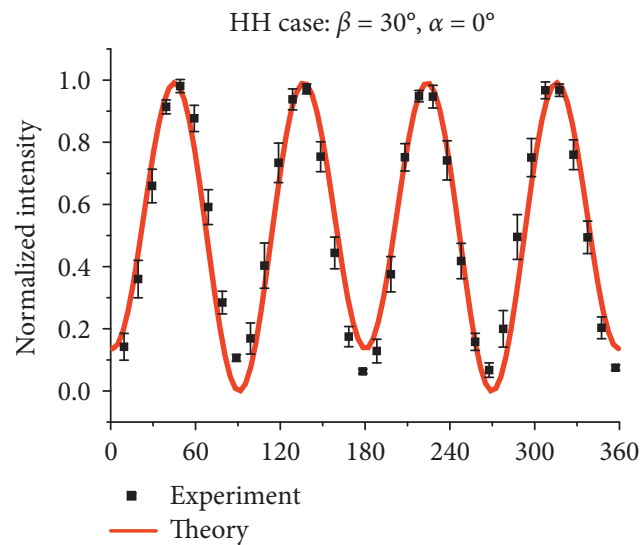

(c)

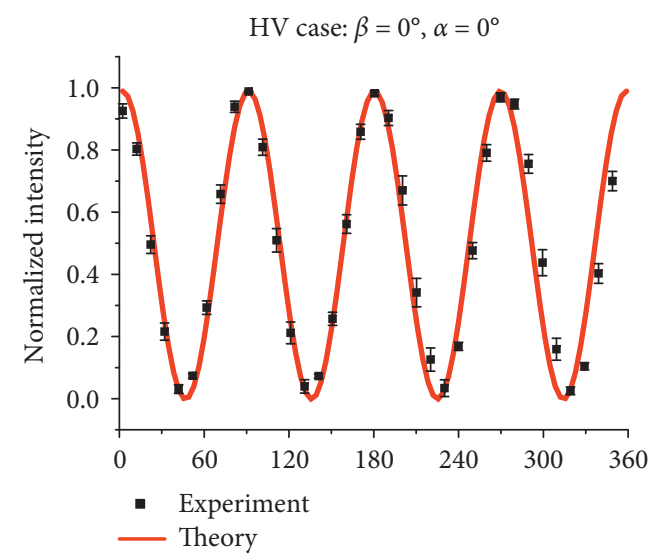

(b)

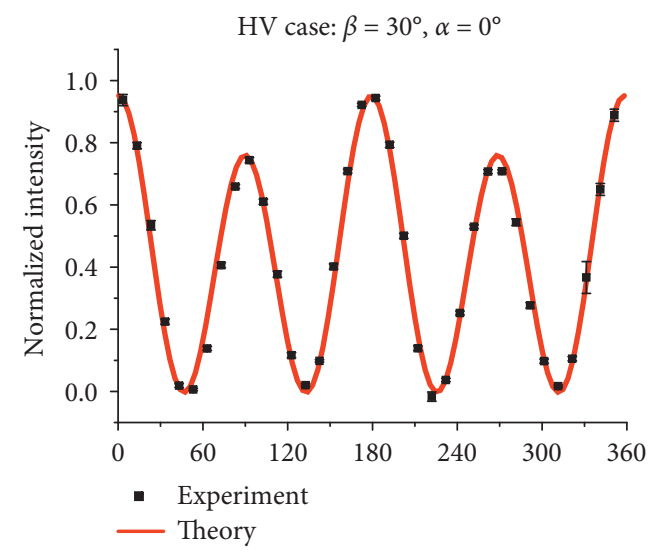

(d)

FIGURE 17: Comparison of experimental results (black solid points) and theoretical results (red solid line) under different geometrical configurations. (a) HH case: $\beta=0^{\circ}, \alpha=0^{\circ}$. (b) HV case: $\beta=0^{\circ}, \alpha=0^{\circ}$. (c) HH case: $\beta=30^{\circ}, \alpha=0^{\circ}$. (d) HV case: $\beta=30^{\circ}, \alpha=0^{\circ}$.

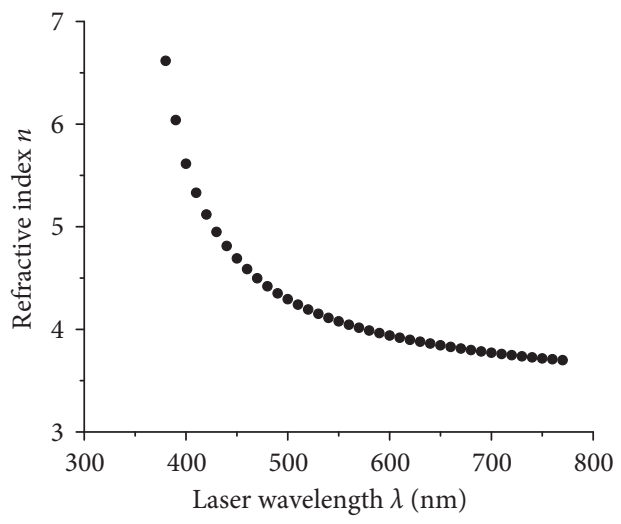

Figure 18: Refractive index of intrinsic silicon at $300 \mathrm{~K}$ (visible light).

illumined in the same cone range, regardless of the backscattering configuration. The model considering the influence of a large NA could be presented as the integral of the Raman intensity caused by the laser in a cone whose inclination angle $\beta \in\left[0^{\circ}, \arcsin (\mathrm{NA})\right]$ and rotation angle $\alpha \in\left[0^{\circ}, 360^{\circ}\right]$. Therefore, the expression of the Raman intensity is presented as shown in (24) when considering the NA:

$$
I_{j} \propto C \int_{0}^{2 \pi} \int_{0}^{\arcsin (N . A .)} \kappa^{2}(\beta)\left|\mathbf{e}_{\mathbf{I}}(\beta) \mathbf{R}_{\mathbf{j}} \mathbf{e}_{\mathbf{S}}(\beta)\right|^{2} \mathrm{~d} \beta \mathrm{d} \alpha,
$$

where $\kappa$ is the energy distribution function of the light spot on the focal plane, which is very dependent on the status of the Raman instruments and the specific parameters of the optical elements, such as the objective lens. 


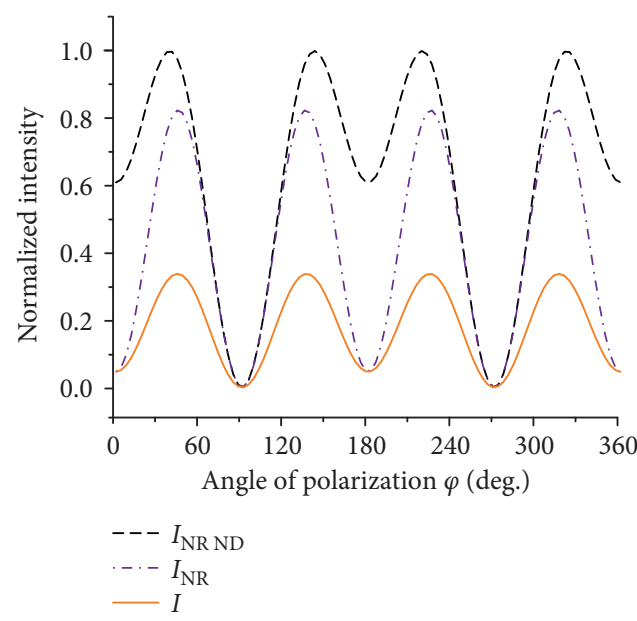

(a)

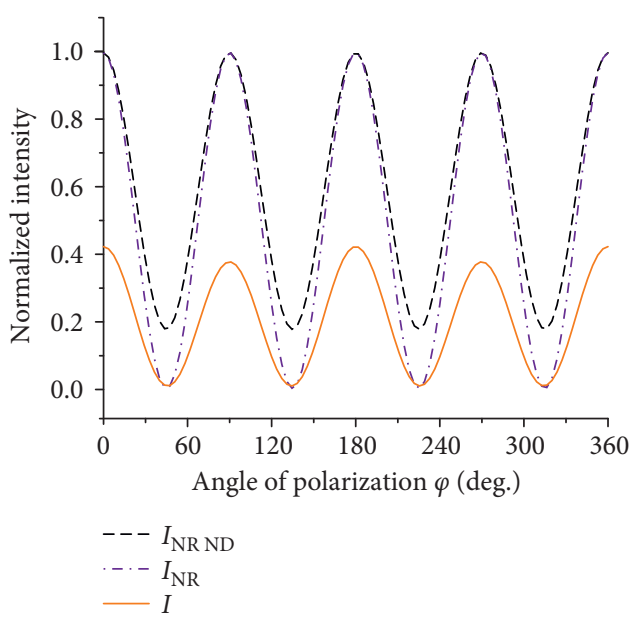

(b)

Figure 19: Raman intensity distribution under the oblique backscattering configuration $\left(\beta_{\mathrm{i}}=\beta_{\mathrm{s}}=30^{\circ}\right)$ for different polarization directions $\varphi$ in the (a) $\mathrm{HH}$ case and (b) HV case considering and not considering the influence of the refractive index and depolarization.

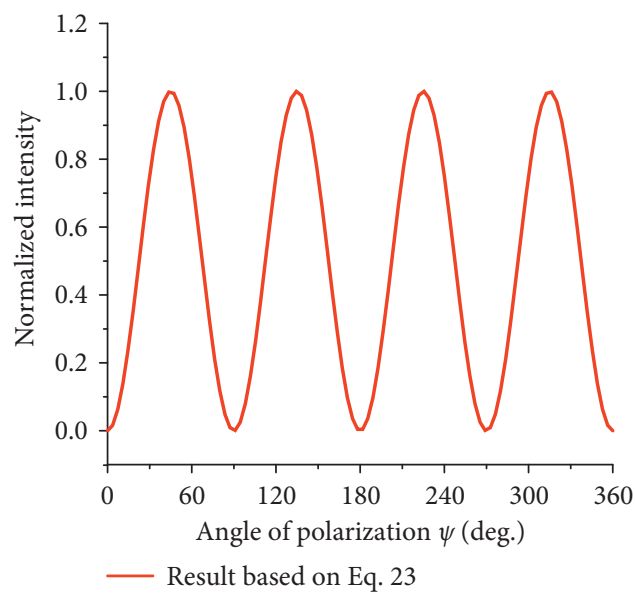

(a)

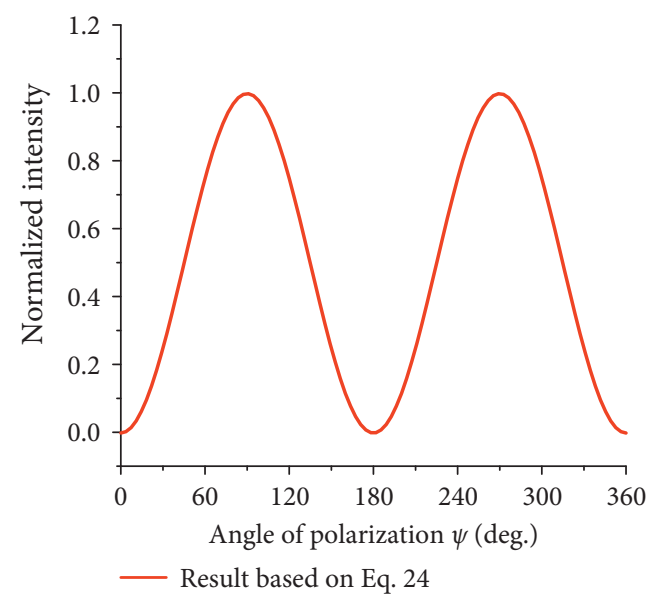

(b)

FIgURe 20: Comparison of the Raman intensities given (a) by (A.3) and (b) by (A.4).

\section{Conclusion}

The Raman intensity shows multivariant trends under different geometrical and polarized configurations and crystal structures. The angular parameters include the inclination angle, polarization directions of the incident laser and the scattered light, and sample rotation angle. In this paper, the theory of the angle-resolved in-axis/off-axis polarized Raman intensity is thoroughly analyzed, and the influence of refraction and depolarization at the air-silicon interface is considered. The phenomena and reasons for the nonstandard sinusoidal curves at different inclination angles are revealed, which is important for analyses of, for example, the crystallographic orientation, Raman tensor calibration, and stress/strain measurement based on angle-resolved in-axis/ off-axis polarized Raman spectroscopy. A general method was established in this work which is suitable for the angleresolved Raman intensity analysis of most crystal materials.

\section{Appendix}

\section{A. Incorrect Concept Definitions}

For a classical vertical backscattering Raman system, $e_{\mathrm{I}}^{\mathrm{z}}=e_{\mathrm{S}}^{\mathrm{z}}=0$. In this case, $e_{\mathrm{I}}^{\mathrm{x}}=e_{\mathrm{S}}^{\mathrm{y}}$ and $e_{\mathrm{I}}^{\mathrm{y}}=-e_{\mathrm{S}}^{\mathrm{x}}$ can be used $[21,27]$, and the dot product of two vectors $e_{\mathrm{I}}$ and $e_{\mathrm{S}}$ is zero. However, in Section 3.1 of that work [8], it is claimed that "when $e_{\mathrm{I}}^{\mathrm{x}}=e_{\mathrm{S}}^{x}$ and $e_{\mathrm{I}}^{y}=e_{\mathrm{S}}^{y}$ and $e_{\mathrm{I}}^{z}=e_{\mathrm{S}}^{z}$, the authors maintain $\mathrm{HH}$ configuration, and if $e_{\mathrm{I}}^{x}=e_{\mathrm{S}}^{y}$ and $e_{\mathrm{I}}^{y}=e_{\mathrm{S}}^{x}$ and $e_{\mathrm{I}}^{z}=e_{\mathrm{S}}^{z}$, the authors maintain HV configuration." There is a mistake in the definition of the HV configuration. Moreover, this definition is not applicable when the Raman spectroscopy measurement is under the geomatical configuration of oblique backscattering. Because $e_{\mathrm{I}}^{z} \neq 0$ and $e_{\mathrm{S}}^{z} \neq 0, e_{\mathrm{I}}^{x}=e_{\mathrm{S}}^{y}$ and $e_{\mathrm{I}}^{y}=-e_{\mathrm{S}}^{x}$ are no longer applicable. In this case, the definition should be based on the actual situation and satisfy the mathematical vertical relationship of the vector; that is, $e_{\mathrm{I}} \cdot e_{\mathrm{S}}=0$. This would lead 
to the definition error of the initial polarization vector no longer being applicable in the following text.

\section{B. Inconsistent Context Definition}

As noted in Section 3.1 [8], "when $e_{\mathrm{I}}^{x}=e_{\mathrm{S}}^{x}$ and $e_{\mathrm{I}}^{y}=e_{\mathrm{S}}^{y}$ and $e_{\mathrm{I}}^{z}=e_{\mathrm{S}}^{z}$, the authors maintain $\mathrm{HH}$ configuration." The $\mathrm{HH}$ configuration means that the vectors $\mathbf{e}_{\mathrm{I}}$ and $\mathbf{e}_{\mathrm{S}}$ satisfy $e_{\mathrm{I}}^{x}=e_{\mathrm{S}}^{x}$, $e_{\mathrm{I}}^{y}=e_{\mathrm{S}}^{y}$, and $e_{\mathrm{I}}^{z}=e_{\mathrm{S}}^{z}$. In this case, the vector $\mathrm{e}_{\mathrm{S}}$ in (11) of Chapter 3.1.1 [8] should be

$$
\mathbf{e}_{\mathbf{S}}=\mathbf{e}_{\mathbf{I}}=\left[\begin{array}{lll}
\cos \psi & \sin \psi & 0
\end{array}\right]^{T},
$$

instead of

$$
\mathbf{e}_{\mathbf{S}}=\left[\begin{array}{lll}
1 & 0 & 0
\end{array}\right]^{T},
$$

where $\psi$ in (A.1) is $\varphi$ in this work.

As calculated using the vectors $\mathbf{e}_{\mathbf{I}}$ and $\mathbf{e}_{\mathbf{S}}$ in (A.1), the relevant TO modes and LO mode should be

$$
\begin{gathered}
\mathrm{TO}_{1} \text { mode: } I_{x} \propto\left|\mathbf{e}_{\mathbf{I}} \mathbf{R}_{\mathbf{x}} \mathbf{e}_{\mathbf{S}}\right|^{2}=\left|\left[\begin{array}{lll}
\cos \psi & \sin \psi & 0
\end{array}\right]\left[\begin{array}{lll}
0 & 0 & 0 \\
0 & 0 & b \\
0 & b & 0
\end{array}\right]\left[\begin{array}{c}
\cos \psi \\
\sin \psi \\
0
\end{array}\right]\right|^{2}=0, \\
\mathrm{TO}_{2} \text { mode: } I_{y} \propto\left|\mathbf{e}_{\mathbf{I}} \mathbf{R}_{\mathbf{y}} \mathbf{e}_{\mathbf{S}}\right|^{2}=\left|\left[\begin{array}{lll}
\cos \psi & \sin \psi & 0
\end{array}\right]\left[\begin{array}{lll}
0 & 0 & b \\
0 & 0 & 0 \\
b & 0 & 0
\end{array}\right]\left[\begin{array}{c}
\cos \psi \\
\sin \psi \\
0
\end{array}\right]\right|^{2}=0, \\
\text { LO mode: } I_{z} \propto\left|\mathbf{e}_{\mathbf{I}} \mathbf{R}_{\mathbf{z}} \mathbf{e}_{\mathbf{S}}\right|^{2}=\left|\left[\begin{array}{lll}
\cos \psi & \sin \psi & 0
\end{array}\right]\left[\begin{array}{lll}
0 & b & 0 \\
b & 0 & 0 \\
0 & 0 & 0
\end{array}\right]\left[\begin{array}{c}
\cos \psi \\
\sin \psi \\
0
\end{array}\right]\right|^{2}=b^{2} \sin ^{2} 2 \psi .
\end{gathered}
$$

However, as calculated using the vectors $\mathbf{e}_{\mathbf{I}}$ and $\mathbf{e}_{\mathbf{S}}$ given in Chapter 3.1.1, the relevant TO modes and LO mode become

$$
\begin{aligned}
& \text { TO }_{1} \text { mode: } I_{x} \propto\left|\mathbf{e}_{\mathbf{I}} \mathbf{R}_{\mathbf{x}} \mathbf{e}_{\mathbf{S}}\right|^{2}=\left|\left[\begin{array}{lll}
\cos \psi & \sin \psi & 0
\end{array}\right]\left[\begin{array}{lll}
0 & 0 & 0 \\
0 & 0 & b \\
0 & b & 0
\end{array}\right]\left[\begin{array}{l}
1 \\
0 \\
0
\end{array}\right]\right|^{2}=0, \\
& \mathrm{TO}_{2} \text { mode: } I_{y} \propto\left|\mathbf{e}_{\mathbf{I}} \mathbf{R}_{\mathbf{y}} \mathbf{e}_{\mathbf{S}}\right|^{2}=\left|\left[\begin{array}{lll}
\cos \psi & \sin \psi & 0
\end{array}\right]\left[\begin{array}{lll}
0 & 0 & b \\
0 & 0 & 0 \\
b & 0 & 0
\end{array}\right]\left[\begin{array}{l}
1 \\
0 \\
0
\end{array}\right]\right|^{2}=0, \\
& \text { LO mode: } I_{z} \propto\left|\mathbf{e}_{\mathbf{I}} \mathbf{R}_{\mathbf{z}} \mathbf{e}_{\mathbf{S}}\right|^{2}=\left|\left[\begin{array}{lll}
\cos \psi & \sin \psi & 0
\end{array}\right]\left[\begin{array}{lll}
0 & b & 0 \\
b & 0 & 0 \\
0 & 0 & 0
\end{array}\right]\left[\begin{array}{l}
1 \\
0 \\
0
\end{array}\right]\right|^{2}=b^{2} \sin ^{2} \psi .
\end{aligned}
$$

According to (A.3) and (A.4), the $\psi$-intensity curves of the LO mode are as shown in Figures 20(a) and 20(b), respectively. The result shown in Figure 20(a) is similar to both the theoretical result shown in Figure 3(a) and the experimental result given in Figure 17(a) but is not similar to the one shown in Figure 20(b), namely, that according to (A.4). Therefore, the correct equations of the Raman intensity in the HH configuration are those in (A.3). The errors induced by the inconsistent definition of $\mathbf{e}_{\mathbf{s}}$ in the $\mathrm{HH}$ configuration caused the mistake in the intensity analysis.

\section{Inconsistency of the Experimental Results with the Theoretical Results}

In Section 4 of that work [8], the experimental results are not consistent with the theoretical results given in the same paper. Moreover, their experimental results are nearly the same as the theoretical result in this work (see (11)) shown in Figure 17(a), which further proves that the theoretical results in this work are correct and that those in the paper by Ramabadran and Roughani [8] are incorrect. 


\section{Data Availability}

The data that support the findings of this study are available from the corresponding author upon reasonable request.

\section{Conflicts of Interest}

The authors declare that there are no conflicts of interest regarding the publication of this paper.

\section{Acknowledgments}

This research was funded by the National Natural Science Foundation of China (Grant nos. 11890682, 11827802, and 12125203).

\section{References}

[1] T. Ma, J. Wang, and D. Li, "Curvature-modulated Si spherical cap-like structure fabricated by multistep ring edge etching," Micromachines, vol. 11, no. 8, 764 pages, 2020.

[2] S. Ke, D. Li, and S. Chen, "A review: wafer bonding of Si-based semiconductors," Journal of Physics D Applied Physics, vol. 53, no. 32, Article ID 323001, 2020.

[3] L. A. Lyon, C. D. Keating, A. P. Fox, E. Baker, and M. J. Natan, "Raman spectroscopy," Analytical Chemistry, vol. 70, no. 12, pp. 341R-361R, 1998.

[4] L. A. Nafie, "Recent advances in linear and non-linear Raman spectroscopy. Part XI," Journal of Raman Spectroscopy, vol. 48, no. 12, pp. 1692-1717, 2017.

[5] C. Xu, T. Xue, J. Guo et al., "An experimental investigation on the tangential interfacial properties of graphene: size effect," Materials Letters, vol. 161, pp. 755-758, 2015.

[6] R. Li, Y. Shang, H. Xing, H. Wang, M. Sun, and W. Qiu, "Orientation identification of the black phosphorus with different thickness based on B-2g mode using a micro-Raman spectroscope under a nonanalyzer configuration," Materials, vol. 13, no. 23, Article ID 5572, 2020.

[7] L. Ma, H. Xing, Q. Ding, Y. Han, Q. Li, and W. Qiu, “Analysis of residual stress around a Berkovich nano-indentation by micro-Raman spectroscopy," AIP Advances, vol. 9, no. 1, Article ID 015010, 2019.

[8] U. Ramabadran and B. Roughani, "Intensity analysis of polarized Raman spectra for off axis single crystal silicon," Materials Science and Engineering: B, vol. 230, pp. 31-42, 2018.

[9] Y. Chang, A. Xiao, R. Li et al., "Angle-resolved intensity of polarized micro-Raman spectroscopy for $4 \mathrm{H}-\mathrm{SiC}$," Crystal, vol. 11, no. 6, 626 pages, 2021.

[10] W. Dou, C. Xu, J. Guo et al., "Interfacial mechanical properties of double-layer graphene with consideration of the effect of stacking mode," ACS Applied Materials \& Interfaces, vol. 10, no. 51, pp. 44941-44949, 2018.

[11] R. Gudaitis, A. Lazauskas, A. Jankauskas, and S. Meskinis, "Catalyst-less and transfer-less synthesis of graphene on $\mathrm{Si}(100)$ using direct microwave plasma enhanced chemical vapor deposition and protective enclosures," Materials, vol. 13, no. 24, Article ID 5630, 2020.

[12] W. Qiu, Y. Kang, Q. Li, Z. Lei, and Q. Qin, "Experimental analysis for the effect of dynamic capillarity on stress transformation in porous silicon," Applied Physics Letters, vol. 92, no. 4, 397 pages, 2008.
[13] Z. Lei, F. Qin, Q. Fang, R. Bai, W. Qiu, and X. Chen, "Full-field fabric stress mapping by micro Raman spectroscopy in a yarn push-out test," Applied Optics, vol. 57, no. 4, 924 pages, 2018.

[14] M. M. Rahaman, T. Imai, T. Sakamoto, S. Tsukada, and S. Kojima, "Fano resonance of Li-doped KTa1xNbxO3 single crystals studied by Raman scattering," Scientific Reports, vol. 6, no. 1, Article ID 23898, 2016.

[15] C. Sun, Y. Wang, H. Gu et al., "Interfacial coupled design of epitaxial Graphene@SiC Schottky junction with built-in electric field for high-performance anodes of lithium ion batteries," Nano Energy, vol. 77, Article ID 105092, 2020.

[16] R. Loudon, "The Raman effect in crystals," Advances in Physics, vol. 13, no. 52, pp. 423-482, 1964.

[17] E. Anastassakis, A. Pinczuk, E. Burstein, F. Pollak, and M. Cardona, "Effect of static uniaxial stress on the Raman spectrum of silicon," Solid State Communications, vol. 8, no. 2, pp. 133-138, 1993.

[18] I. D. Wolf, "Stress measurements in Si microelectronics devices using Raman spectroscopy," Journal of Raman Spectroscopy, vol. 30, no. 10, pp. 877-883, 1999.

[19] I. De Wolf, H. E. Maes, and S. K. Jones, "Stress measurements in silicon devices through Raman spectroscopy: bridging the gap between theory and experiment," Journal of Applied Physics, vol. 79, no. 9, pp. 7148-7156, 1996.

[20] W. Qiu and Y.-L. Kang, "Mechanical behavior study of microdevice and nanomaterials by Raman spectroscopy: a review," Chinese Science Bulletin, vol. 59, no. 23, pp. 2811-2824, 2014.

[21] L. Ma, X. Fan, and W. Qiu, "Polarized Raman spectroscopystress relationship considering shear stress effect," Optics Letters, vol. 44, no. 19, pp. 4682-4685, 2019.

[22] G. H. Loechelt, N. G. Cave, and J. Menéndez, "Polarized offaxis Raman spectroscopy: a technique for measuring stress tensors in semiconductors," Journal of Applied Physics, vol. 86, no. 11, pp. 6164-6180, 1999.

[23] Y. Takahashi, L. Puppulin, W. Zhu, and G. Pezzotti, "Raman tensor analysis of ultra-high molecular weight polyethylene and its application to study retrieved hip joint components," Acta Biomaterialia, vol. 6, no. 9, pp. 3583-3594, 2010.

[24] R. C. Jones, "A new calculus for the treatment of optical systems VII properties of the N-matrices," Journal of the Optical Society of America, vol. 38, no. 8, pp. 671-685, 1948.

[25] M. Born, P. C. Clemmow, and D. Gabor, Principles of Optics, Pergamon Press, New York, NY, USA, 1959.

[26] M. A. Green, "Self-consistent optical parameters of intrinsic silicon at $300 \mathrm{~K}$ including temperature coefficients," Solar Energy Materials and Solar Cells, vol. 92, no. 11, pp. 1305-1310, 2015.

[27] L. Ma, H. Xing, Q. Li, J. Wang, and W. Qiu, "Raman stress measurement of crystalline silicon desensitizes shear stress: only on $\{001\}$ crystal plane," Japanese Journal of Applied Physics, vol. 57, no. 8, Article ID 080307, 2018. 